\title{
Coherent and incoherent theories for photosynthetic energy transfer
}

\author{
Ming-Jie Tao, ${ }^{a, b, *}$ Na-Na Zhang, ${ }^{b, *}$ Peng-Yu Wen,${ }^{b}$ Fu-Guo Deng,,${ }^{b, c}$ Qing Ai,${ }^{b, \dagger}$ Gui-Lu Long ${ }^{a, \ddagger}$ \\ ${ }^{a}$ Department of Physics, Tsinghua University, Beijing 100084, China \\ ${ }^{b}$ Department of Physics, Applied Optics Beijing Area Major Laboratory, Beijing Normal University, Beijing 100875, China \\ ${ }^{c}$ NAAM-Research group, Department of Mathematics, Faculty of Science, \\ King Abdulaziz University, Jeddah 21589, Saudi Arabia
}

(Dated: January 24, 2020)

\begin{abstract}
There is a remarkable characteristic of photosynthesis in nature, that is, the energy transfer efficiency is close to $100 \%$. Recently, due to the rapid progress made in the experimental techniques, quantum coherent effects have been experimentally demonstrated. Traditionally, the incoherent theories are capable of calculating the energy transfer efficiency, e.g., (generalized) Förster theory and modified Redfield theory (MRT). However, in order to describe the quantum coherent effects in photosynthesis, one has to exploit coherent theories, such as hierarchical equation of motion (HEOM), quantum path integral, coherent modified Redfield theory (CMRT), small-polaron quantum master equation, and general Bloch-Redfield theory in addition to the Redfield theory. Here, we summarize the main points of the above approaches, which might be beneficial to the quantum simulation of quantum dynamics of exciton energy transfer (EET) in natural photosynthesis, and shed light on the design of artificial light-harvesting devices.
\end{abstract}

PACS numbers:

Keywords: Förster theory, modified Redfield theory, hierarchical equation of motion, coherent modified Redfield theory, small-polaron quantum master equation, general BlochRedfield theory.

\section{Introduction}

In the past two decades, quantum coherence phenomena have been demonstrated to exist and even support the physiological processes in biology [1, 2], e.g., avian navigation and exciton energy transfer (EET) in natural photosynthesis. The former has been indirectly confirmed by a number of interesting experiments [3-5]. And the entanglement between the pair of natural qubits, radicals, was shown to last over milliseconds at the ambient condition, which is significantly longer than those artificial quantum systems at a sufficientlylow temperature and vacuum [6-8] underlying physical mechanism, Ritz et al. [9] proposed the radical-pair hypothesis to describe how birds utilize the weak geomagnetic field for navigation. In the radical-pair hypothesis, the inter-conversion between the spin-singlet and triplet states induced by the geomagnetic field and the local field posed by the nuclear spins results in distinguishable products of chemical reaction. This kind of magnetic-field sensitive chemical reactions can be well described by the generalized Holstein model with spin degrees of freedom taken into consideration [10]. Since the detection sensitivity subtly depends on the interplay of the nuclear spins and geomagnetic field, it was suggested that when there is quantum phase transition in the nuclear spins [11-13], the sensitivity can be significantly increased by quantum criticality [14].

\footnotetext{
*These authors contributed equally to this work

$\dagger$ aiqing@bnu.edu.cn

†gllong@tsinghua.edu.cn
}

On the other hand, photosynthesis is a complex biochemical reaction process. It is well known that the process of photosynthesis mainly includes four aspects, namely, primary reactions, electron transfer, photophosphorylation and carbon dioxide fixation [15]. In the process of primary reaction, one of the peripheral light-harvesting antennas absorbs a photon of sunlight, and then the pigments transfer the captured energy to the reaction center. In the reaction center, electrons are transferred and a potential difference is generated to drive the subsequent biochemical reactions. The processes of energy and electron transfer are ultrafast, both of them occurring around $10^{-12}$ seconds. The excitation energy is delivered to the reaction center on a time scale of 30 picoseconds, and subsequent electron transfer in about 3 picoseconds. Due to nearly $100 \%$ quantum efficiency, the energy transfer mechanism has aroused widespread interests. One of the current research hotspots is the study of the physical mechanism of EET in photosynthesis. In the late 1980s, the molecular structure of the protein complex of the purple bacteriological reaction center was obtained [16]. Subsequently, some other molecular structures of reaction centers and light-harvesting antenna protein complexes were generally determined [17-21]. The increased understanding of the molecular structures of photosynthesis is helpful to the development of artificial lightharvesting devices [22].

Up to now, the study on EET has been made much progress [18-42]. A series of interesting experiments point out that quantum coherence may play an important role in the process of EET in photosynthesis. For example, it has been demonstrated that exciton delocalization optimizes the energy transfer from B800 ring to B850 ring in LH2 [21]. In addition, it seems that there is some relationship between quantum coherence and the high efficiency of EET [25]. Moreover, Caruso et al. [26] studied the relation between the degree of entanglement and EET efficiency in photosynthesis. They believed that the EET in photosynthesis is realized by the interplay of coherent and incoherent processes. Sarovar et al. 
[27] found that the coherence between pigments almost exists in the whole process of EET. Fassioli and Olaya-Castro [28] showed that there is an inverse relationship between the quantum efficiency and the average entanglement between distant donor sites in FMO complex.

As photosynthetic complexes inevitably interact with the surrounding environment, some scientists have also begun to study the quantum noise effects on EET efficiency. After studying the impact of environment on EET, Leon-Montiel and Torres [30] found that even in purely-classical systems, the efficiency of EET can be improved by adjusting the external environment. Mohseni et al. [31-33] proposed the concept of environmental-noise-assisted EET in the study of FMO photosynthetic complexes. Cao and Silbey [34] pointed out that the optimal exciton trapping scheme can be achieved by choosing the appropriate decoherence rate and exciton trapping rate. Plenio and his collaborators [26] revealed that there is a certain correlation among the EET efficiency, the energy detuning and dephasing. Although the above research results were obtained using numerical methods, these results have revealed that adjusting the environmental noise can improve the EET efficiency.

In the above continuous study of EET in photosynthesis, many theoretical methods have been proposed. They can be divided into coherent and incoherent theories. Traditionally, the incoherent theories, e.g., Förster theory and MRT, were employed to calculate the energy transfer efficiency. In 2007, Fleming's group [25] first observed quantum coherence in FMO complexes at low temperatures $(T=77 \mathrm{~K})$ for 660 fs. This discovery made people more aware that the energy transfer among pigments does not fully follow Förster theory. For the coherent theories, there are hierarchical equation of motion (HEOM), quantum path integrals, coherent modified Redfield theory (CMRT), small-polaron quantum master equation, and general Bloch-Redfield theory. This paper mainly introduces these incoherent and coherent theories, and analyses the applicable conditions, advantages and disadvantages of each theory.

\section{Incoherent theories for photosynthesis}

In this section, we will mainly introduce the incoherent theories, which are efficient for calculating the efficiency of EET in photosynthesis [43-46].

\section{A. Förster theory}

Initially, people used Förster theory to describe energy transfer in photosynthesis. The condition of Förster theory is that the distance between molecules is long enough in the process of energy transfer. For a donor (D) and an acceptor (A), the rate of EET between them can be described by the Förster theory as [47, 48]

$$
k_{\mathrm{D} \rightarrow \mathrm{A}}^{\mathrm{F}}=\frac{J_{\mathrm{DA}}^{2}}{2 \pi \hbar^{2}} \int_{-\infty}^{\infty} \mathrm{d} \omega E_{\mathrm{D}}(\omega) I_{\mathrm{A}}(\omega),
$$

where $\omega$ is the frequency and $\hbar$ is the Planck constant, $J_{\mathrm{DA}}$ represents the Coulomb coupling between D and A, which is inversely proportional to the cubic power of the distance between D and A. $E_{\mathrm{D}}(\omega)$ and $I_{\mathrm{A}}(\omega)$ represent the fluorescence and the absorption spectrum respectively, which can be directly measured by the experiment and can also be calculated from the theory as

$$
\begin{gathered}
E_{\mathrm{D}}(\omega)=\int_{-\infty}^{\infty} \mathrm{d} t \mathrm{e}^{-\mathrm{i} \omega t} \operatorname{Tr}_{\mathrm{B}}\left(\rho_{\mathrm{D}}^{\mathrm{e}} \mathrm{e}^{-\mathrm{i} \mathrm{H}_{\mathrm{D}}^{\mathrm{e}} \mathrm{t} / \mathrm{h}} \mathrm{e}^{\mathrm{i}\left(\varepsilon_{\mathrm{D}}+\mathrm{H}_{\mathrm{D}}^{\mathrm{g}}\right) t / \hbar}\right), \\
I_{\mathrm{A}}(\omega)=\int_{-\infty}^{\infty} \mathrm{d} t \mathrm{e}^{\mathrm{i} \omega t} \operatorname{Tr}_{\mathrm{B}}\left(\rho_{\mathrm{A}}^{\mathrm{e}} \mathrm{e}^{\mathrm{i} H_{\mathrm{A}}^{\mathrm{e}} t / \hbar} \mathrm{e}^{-\mathrm{i}\left(\varepsilon_{\mathrm{A}}+H_{\mathrm{A}}^{\mathrm{g}}\right) t / \hbar}\right) .
\end{gathered}
$$

Here $\varepsilon_{\mathrm{D}}$ and $\varepsilon_{\mathrm{A}}$ are excited-state energies of $\mathrm{D}$ and $\mathrm{A}$, respectively.

$$
\begin{aligned}
H_{\mathrm{D}}^{\mathrm{g}} & =\sum_{j} \omega_{j} a_{j}^{\dagger} a_{j}, \\
H_{\mathrm{A}}^{\mathrm{g}} & =\sum_{j} \omega_{j} b_{j}^{\dagger} b_{j},
\end{aligned}
$$

are the Hamiltonians corresponding to the baths of D and A when they are in the ground states, respectively. $a_{j}\left(b_{j}\right)$ is the annihilation operator of donor's (acceptor's) $j$ th harmonic oscillator with frequency $\omega_{j}$.

$$
\begin{aligned}
& H_{\mathrm{D}}^{\mathrm{e}}=\sum_{j} \omega_{j} a_{j}^{\dagger} a_{j}+\sum_{j} g_{j}^{\mathrm{D}}\left(a_{j}^{\dagger}+a_{j}\right), \\
& H_{\mathrm{A}}^{\mathrm{e}}=\sum_{j} \omega_{j} b_{j}^{\dagger} b_{j}+\sum_{j} g_{j}^{\mathrm{A}}\left(b_{j}^{\dagger}+b_{j}\right),
\end{aligned}
$$

are the Hamiltonians of D's and A's bath when they are at the excited state respectively, where $g_{j}^{\mathrm{D}}\left(g_{j}^{\mathrm{A}}\right)$ is the coupling strength with D's (A's) $j$ th mode. $\rho_{\mathrm{D}}^{\mathrm{e}}=\mathrm{e}^{-\beta H_{\mathrm{D}}^{\mathrm{e}}} / \operatorname{Tr}_{\mathrm{B}} \mathrm{e}^{-\beta \mathrm{H}_{\mathrm{D}}^{\mathrm{e}}}$ $\left(\rho_{\mathrm{A}}^{\mathrm{e}}=\mathrm{e}^{-\beta H_{\mathrm{A}}^{\mathrm{e}}} / \operatorname{Tr}_{\mathrm{B}} \mathrm{e}^{-\beta \mathrm{H}_{\mathrm{A}}^{\mathrm{e}}}\right)$ is the D's (A's) bath's density matrix at the thermal equilibrium, where $\beta=k_{\mathrm{B}} T$ is the inverse temperature with $k_{\mathrm{B}}$ and $T$ being the Boltzmann constant and temperature, respectively.

Förster theory has been widely used in practical experiments and theories for studying energy transfer at the early stages. However, it is worth noting that the formula is derived under some approximations. Because it is assumed that the intra-system coupling $J_{\mathrm{DA}}$ is much smaller than the systembath couplings $g_{j}^{\mathrm{D}}$ and $g_{j}^{\mathrm{A}}$. Furthermore, for the sake of simplicity, the intra-system coupling $J_{\mathrm{DA}}$ is generally calculated by the dipole-dipole approximation. Therefore, it may break down when the distance between $\mathrm{D}$ and $\mathrm{A}$ is relative small as compared to charge distribution within the $\mathrm{D}(\mathrm{A})$.

\section{B. Generalized Förster theory}

In the preceding subsection, we have introduced Förster theory. It was developed for calculating the rate of EET between a donor (D) and an acceptor (A). However, for a cluster formed by strong couplings of several chromophores, Eq. (1) can not accurately simulate population dynamics. This is mainly because the original Förster theory treats the internal couplings of the system as a perturbation. Therefore, when 
there are some clusters, the original Förster theory should be generalized to describe the EET in a multichromophoric system [49, 50].

The spectral overlap in Eq. (1) is a central feature of the Förster theory. Note that there is no bath's degree of freedom simultaneously coupled to D and A. Under this assumption, the Förster theory can be generalized.

For a multichromophoric system, D and A are composed of $\mathrm{D}_{j}\left(j=1, \ldots, N_{\mathrm{D}}\right)$ and $\mathrm{A}_{k}\left(k=1, \ldots, N_{\mathrm{A}}\right)$, respectively [49]. We suppose that the exciton Hamiltonians is

$$
\begin{aligned}
& H_{\mathrm{D}, 0}^{\mathrm{e}}=\sum_{j=1}^{N_{\mathrm{D}}} \varepsilon_{\mathrm{D}_{j}}\left|\mathrm{D}_{j}\right\rangle\left\langle\mathrm{D}_{j}\left|+\sum_{j \neq j^{\prime}} \Delta_{j j^{\prime}}^{\mathrm{D}}\right| \mathrm{D}_{j}\right\rangle\left\langle\mathrm{D}_{j^{\prime}}\right|, \\
& H_{\mathrm{A}, 0}^{\mathrm{e}}=\sum_{k=1}^{N_{\mathrm{A}}} \varepsilon_{\mathrm{A}_{k}}\left|\mathrm{~A}_{k}\right\rangle\left\langle\mathrm{A}_{k}\left|+\sum_{k \neq k^{\prime}} \Delta_{k k^{\prime}}^{\mathrm{A}}\right| \mathrm{A}_{k}\right\rangle\left\langle\mathrm{A}_{k^{\prime}}\right|,
\end{aligned}
$$

where $\varepsilon_{\mathrm{D}_{j}}\left(\varepsilon_{\mathrm{A}_{k}}\right)$ is the energy of the excitation state $\left|\mathrm{D}_{j}\right\rangle\left(\left|\mathrm{A}_{k}\right\rangle\right)$, and $\Delta_{j j^{\prime}}^{\mathrm{D}}\left(\Delta_{k k^{\prime}}^{\mathrm{A}}\right)$ is the electronic coupling between $\left|\mathrm{D}_{j}\right\rangle$ and $\left|\mathrm{D}_{j^{\prime}}\right\rangle\left(\left|\mathrm{A}_{k}\right\rangle\right.$ and $\left.\left|\mathrm{A}_{k^{\prime}}\right\rangle\right)$. In addition to the above electronic states, all other degrees of freedom will be treated as bath. If each $\left|D_{j}\right\rangle$ is coupled to a bath operator $\mathrm{B}_{\mathrm{D}_{\mathrm{j}}}$, the total Hamiltonian for excited D can be expressed as $H_{\mathrm{D}}^{\mathrm{e}}=$ $H_{\mathrm{D}, 0}^{\mathrm{e}}+\sum_{j=1}^{N_{\mathrm{D}}} B_{\mathrm{D}_{j}}\left|\mathrm{D}_{j}\right\rangle\left\langle\mathrm{D}_{j}\right|+H_{\mathrm{D}}^{\mathrm{g}}$. Likewise, the total Hamiltonian for excited $\mathrm{A}$ is $H_{\mathrm{A}}^{\mathrm{e}}=H_{\mathrm{A}, 0}^{\mathrm{e}}+\sum_{k=1}^{N_{\mathrm{A}}} B_{\mathrm{A}_{k}}\left|\mathrm{~A}_{k}\right\rangle\left\langle\mathrm{A}_{k}\right|+H_{\mathrm{A}}^{\mathrm{g}}$. For the assumption that there is no given bath mode couples to both $D$ and $A$, it can be equivalent to $\left[H_{\mathrm{D}}^{\mathrm{g}}, H_{\mathrm{A}}^{\mathrm{g}}\right]=$ $\left[H_{\mathrm{D}}^{\mathrm{g}}, B_{\mathrm{A}_{k}}\right]=\left[H_{\mathrm{A}}^{\mathrm{g}}, B_{\mathrm{D}_{j}}\right]=0$. For the multichromophoric system, $N_{\mathrm{D}} \times N_{\mathrm{A}}$ terms contribute to the coupling Hamiltonian, $H_{c}=\sum_{j=1}^{N_{\mathrm{D}}} \sum_{k=1}^{N_{\mathrm{A}}} J_{j k}\left(\left|\mathrm{D}_{j}\right\rangle\left\langle\mathrm{A}_{k}|+| \mathrm{A}_{k}\right\rangle\left\langle\mathrm{D}_{j}\right|\right)$, where $J_{j k}$ is the dipole-dipole interaction between $\mathrm{D}_{j}$ and $\mathrm{A}_{k}$.

Starting from the Fermi's golden rule [51, 52], we can obtain the multichromophoric Förster rate from the cluster of D to the cluster of $\mathrm{A}$, which is

$$
k^{\mathrm{MC}}(t)=\sum_{j^{\prime} j^{\prime \prime}} \sum_{k^{\prime} k^{\prime \prime}} \frac{J_{j^{\prime} k^{\prime}} J_{j^{\prime \prime} k^{\prime \prime}}}{2 \pi \hbar^{2}} \int_{-\infty}^{\infty} \mathrm{d} \omega E_{\mathrm{D}}^{j^{\prime \prime} j^{\prime}}(t, \omega) I_{\mathrm{A}}^{k^{\prime} k^{\prime \prime}}(\omega),
$$

where

$$
\begin{gathered}
E_{\mathrm{D}}^{j^{\prime \prime} j^{\prime}}(t, \omega) \equiv 2 \operatorname{Re}\left[\int _ { 0 } ^ { t } \mathrm { d } t ^ { \prime } \mathrm { e } ^ { - \mathrm { i } \omega t ^ { \prime } } \operatorname { T r } _ { \mathrm { D } } \left\{\mathrm{e}^{-\mathrm{i} H_{\mathrm{D}}^{\mathrm{g}} t^{\prime} / \hbar}\right.\right. \\
\left.\left.\times\left\langle\mathrm{D}_{j}\left|\mathrm{e}^{-\mathrm{i} H_{\mathrm{D}}^{e}\left(t-t^{\prime}\right) / \hbar}\right| \mathrm{D}_{\hat{\mathbf{e}}}\right\rangle\left\langle\mathrm{D}_{\hat{\mathrm{e}}}\left|\rho_{\mathrm{D}}^{\mathrm{g}} \mathrm{e}^{\mathrm{i} H_{\mathrm{D}}^{e} t / \hbar}\right| \mathrm{D}_{j^{\prime}}\right\rangle\right\}\right], \\
I_{\mathrm{A}}^{k^{\prime} k^{\prime \prime}}(\omega) \equiv \int_{-\infty}^{\infty} \mathrm{d} t \mathrm{e}^{\mathrm{i} \omega t} \operatorname{Tr}_{\mathrm{A}}\left\{\mathrm{e}^{\mathrm{i} H_{\AA}^{\mathrm{g}} t / \hbar}\left\langle\mathrm{A}_{k^{\prime}}\left|\mathrm{e}^{-\mathrm{i} H_{\AA}^{e} t / \hbar}\right| \mathrm{A}_{k^{\prime \prime}}\right\rangle \rho_{\mathrm{A}}^{\mathrm{g}}\right\},
\end{gathered}
$$

are the matrix elements of $\mathbf{E}_{\mathrm{D}}(t, \omega)$ and $\mathbf{I}_{\mathrm{A}}(\omega)$. Here $\operatorname{Tr}_{\mu}$ represents the trace over all the bath degrees of freedom for $\mu=\mathrm{D}$, A. For $t<0$, the total system is in the ground state represented by $\rho_{\mathrm{D}}^{\mathrm{g}} \rho_{\mathrm{A}}^{\mathrm{g}}$. If the appropriate pulse is selected to excite $\mathrm{D}$ at $t=0$, the total density operator will be changed to $\rho(0)=\left|\mathrm{D}_{\hat{\mathbf{e}}}\right\rangle\left\langle\mathrm{D}_{\hat{\mathbf{e}}}\right| \rho_{\mathrm{D}}^{\mathrm{g}} \rho_{\mathrm{A}}^{\mathrm{g}}$, where $\left|\mathrm{D}_{\hat{\mathbf{e}}}\right\rangle=\mathscr{N} \hat{\mathbf{e}} \dot{\sum}_{j} \mu_{\mathrm{D}_{j}}\left|\mathrm{D}_{j}\right\rangle, \mathscr{N}$ is the normalization constant, $\hat{\mathbf{e}}$ is the polarization vector of the radiation, $\mu_{\mathrm{D}_{j}}$ is the transition dipole of $\left|\mathrm{D}_{j}\right\rangle$.
It has been demonstrated that the entanglement between donor and bath plays an important role in both the emission spectrum and multichromophoric Förster rate [52-54]. The coupling between the initial system and the bath will cause the bath to deviate from equilibrium, which will further complicate the emission spectrum. And this deviation will affect the subsequent dynamic evolution, which makes the calculation of multichromophoric Förster rate more complicated. In Ref. [52], a full 2nd-order cumulant expansion technique is developed to calculate the spectra and multichromophoric Förster rate for both localized and delocalized systems. The full 2nd-order cumulant expansion technique is reliable for the absorption and emission spectra of localized system. Moreover, for both localized and delocalized systems, the multichromophoric Förster rates are close to the exact ones, which can not be obtained by the methods of general second-order theories, such as Redfield theory, MRT, and CMRT. And for the absorption and emission spectra which are expressed in a full 2nd-order cumulant expansion can reduce to the exact results in the monomer case.

\section{Modified Redfield theory}

The Förster theory and its generalization are valid in the regime where the intra-system couplings are much weaker than the system-bath couplings. On the contrary, Redfield theory treats the coupling of exciton and external vibration mode as a perturbation and assumes second-order truncation when the intra-system couplings prevail.

However, in natural photosynthesis, neither conditions hold as the intra-system couplings are comparable to the systembath couplings. Therefore, the Redfield theory is modified to describe the EET in natural photosynthesis [37, 44]]. The MRT adopts the same basis, i.e., the exciton basis, as the Redfield theory. That is to say, when there are closely-connected pigment molecules, the excited state can not be described by the excitation of a single pigment, but its excitation extends to more than one pigment to form an exciton state. The state is a combination of several molecular wave functions, which can be expressed as $|\alpha\rangle=\sum_{n} c_{n}^{\alpha}|n\rangle$, with $|n\rangle$ the excited state of the $n$th pigment.

In this basis, the total Hamiltonian is written as

$$
H=H^{\mathrm{el}}+H^{\mathrm{ph}}+H^{\mathrm{el}-\mathrm{ph}}
$$

where

$$
\begin{aligned}
H^{\mathrm{el}} & =\sum_{\alpha} \varepsilon_{\alpha}|\alpha\rangle\langle\alpha|, \\
H^{\mathrm{ph}}= & \sum_{n, i} \omega_{i} b_{n i}^{\dagger} b_{n i}, \\
H^{\mathrm{el}-\mathrm{ph}}= & \sum_{\alpha, \beta} \sum_{n, i}|\alpha\rangle\langle\beta| c_{n}^{\alpha} c_{n}^{\beta *} g_{n i} \omega_{i}\left(b_{n i}+b_{n i}^{\dagger}\right) \\
& =\sum_{\alpha, \beta}|\alpha\rangle\langle\beta|\left(H^{\mathrm{el}-\mathrm{ph}}\right)_{\alpha \beta} .
\end{aligned}
$$

Here, $\varepsilon_{\alpha}$ is the excitation energy of $|\alpha\rangle, b_{n i}^{\dagger}\left(b_{n i}\right)$ is the creation (annihilation) operator of the $i$ th phonon mode of $n$th pig- 
ment, $\omega_{i}$ is the frequency of the phonon mode, and $g_{n i} \omega_{i}$ is the exciton-phonon coupling constant between the localized electronic excitation on site $n$ and its $i$ th phonon mode. Although here we assume individual bath for each pigment, the general correlated baths can also be described by this model. The reorganization energy of site $n$ can be expressed as $\lambda_{n}=\sum_{i} g_{n i}^{2} \omega_{i}$. Finally, the transformation from the site basis to the exciton basis yields the factor $\sum_{n}^{N} c_{n}^{\alpha} c_{n}^{\beta}$, which can be considered as the overlap between exciton wavefunctions $|\alpha\rangle$ and $|\beta\rangle$.

The MRT aims at dividing the Hamiltonian into a zerothorder Hamiltonian and a perturbation part, which correspond to the diagonal and off-diagonal system-bath couplings in the exciton basis, respectively. The zeroth-order Hamiltonian is

$$
H_{0}=H^{\mathrm{el}}+H^{\mathrm{ph}}+\sum_{\alpha}|\alpha\rangle\langle\alpha| \cdot\left(H^{\mathrm{el}-\mathrm{ph}}\right)_{\alpha \alpha},
$$

and the perturbation Hamiltonian is

$$
V=\sum_{\alpha \neq \beta}|\alpha\rangle\langle\beta| \cdot\left(H^{\mathrm{el}-\mathrm{ph}}\right)_{\alpha \beta} .
$$

Notice that the diagonal part of $H^{\mathrm{el}-\mathrm{ph}}$, cf. Eq. (16), is included in the zeroth-order Hamiltonian while the off-diagonal part of $H^{\mathrm{el}-\mathrm{ph}}$ is exactly the perturbation Hamiltonian. According to second-order perturbation theory, the transfer rate can be expressed as

$$
R_{\alpha \beta}(t)=2 \operatorname{Re} \int_{0}^{t} \mathrm{~d} \tau F_{\beta}^{*}(\tau) A_{\alpha}(\tau) X_{\alpha \beta}(\tau),
$$

where

$$
\begin{aligned}
A_{\alpha}(t) & =\mathrm{e}^{-\mathrm{i} \varepsilon_{\alpha} t-g_{\alpha \alpha \alpha \alpha}(t)}, \\
F_{\alpha}(t) & =\mathrm{e}^{-\mathrm{i}\left(\varepsilon_{\alpha}-2 \lambda_{\alpha \alpha \alpha \alpha}\right) t-g_{\alpha \alpha \alpha \alpha}^{*}(t)} .
\end{aligned}
$$

$A_{\alpha}(t)$ and $F_{\alpha}(t)$ are related to the absorption and emission lineshape, respectively.

$$
\begin{aligned}
X_{\alpha \beta}(t)= & \mathrm{e}^{2\left[g_{\alpha \alpha \beta \beta}(t)+\mathrm{i} \lambda_{\alpha \alpha \beta \beta} t\right]} \\
& \times\left[\ddot{g}_{\beta \alpha \alpha \beta}(t)-\left(\dot{g}_{\beta \alpha \alpha \alpha}(t)-\dot{g}_{\beta \alpha \beta \beta}(t)-2 \mathrm{i} \lambda_{\beta \alpha \beta \beta}\right)\right. \\
& \left.\times\left(\dot{g}_{\alpha \beta \alpha \alpha}(t)-\dot{g}_{\alpha \beta \beta \beta}(t)-2 \mathrm{i} \lambda_{\alpha \beta \beta \beta}\right)\right],
\end{aligned}
$$

which is the perturbation-induced dynamical term. And

$$
\begin{aligned}
g_{\alpha \beta \gamma \delta}(t) & =\sum_{n, m} c_{n}^{\alpha} c_{n}^{\beta *} c_{m}^{\gamma} c_{m}^{\delta *} g_{n m}(t), \\
\lambda_{\alpha \beta \gamma \delta} & =\sum_{n} c_{n}^{\alpha} c_{n}^{\beta *} c_{n}^{\gamma} c_{n}^{\delta *} \lambda_{n} .
\end{aligned}
$$

Here the lineshape function is

$$
\begin{aligned}
g_{n m}(t)= & \int_{0}^{\infty} \mathrm{d} \omega \frac{J_{n m}(\omega)}{\omega^{2}}\left\{\operatorname{coth}\left(\frac{\omega}{2 k_{B} T}\right)[1-\cos (\omega t)]\right. \\
& +\mathrm{i}[\sin (\omega t)-\omega t]\}
\end{aligned}
$$

and the spectral density is

$$
J_{n m}(\omega)=\sum_{i} g_{n i} g_{m i} \omega_{i}^{2} \delta\left(\omega-\omega_{i}\right) .
$$
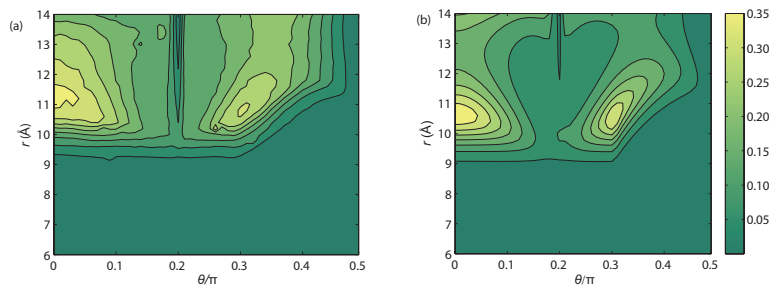

FIG. 1: (Color online) Comparison of EET in a tetramer system [50]: The effective transfer rate is obtained by (a) full simulation with the CMRT, (b) cascade exponential decay model with decay rates calculated by the MRT and generalized Förster theory.

In Ref. [50], in a tetramer system, in addition to the full simulation carried out by the CMRT, the effective transfer rate was also investigated by a cascade exponential decay model with the single decay rate calculated by the MRT within the clusters and generalized Förster theory between the clusters, cf. Fig. 1. It is shown that the simplified calculation captures the effective EET characteristics for a wide parameter range. In this sense, it is reliable to use the incoherent theories to explore the EET efficiency.

It has been shown that the MRT can be successfully used to determine population transfer rates between exciton states beyond the Redfield and Förster regimes, because it takes into consideration multi-phonon processes [44]. However, recent studies have demonstrated that the MRT yields unphysical results for small electronic coupling and energy gap, because it underestimates the dynamical localization due to large reorganization energy [55, 56].

Here, we have summarized the main points of the MRT. Later, by generalizing to describe the quantum dynamics of off-diagonal terms of density matrix, Cheng et al. developed the coherent version of the MRT, i.e., the CMRT, to be capable of simulating the quantum dynamics of the full density matrix, which will be introduced in Sec.D.

\section{Coherent theories for photosynthesis}

Since 2006, there have been a series of interesting experiments [21, 25, 57-59], which implied that coherence might play an important role in the photosynthesis. Since the incoherent theories are only capable of calculating the energy transfer efficiency, theories other than the incoherent ones are needed to consider the effect of coherence on the quantum dynamics. In this section, we summarize the main theories, which can calculate the quantum dynamics of the full density matrix, including both the populations and the off-diagonal terms.

\section{A. Redfield theory}

For open quantum systems, one of the main concerns is to obtain the equation of the system's reduced density matrix by 
eliminating the degrees of freedom of the environment 60 62]. Generally, the quantum dynamics of the reduced density matrix can be obtained from the Liouville equation by the perturbation expansion, e.g., Redfield theory [47].

For an open quantum system, the total Hamiltonian $\mathbf{H}_{\mathrm{t}}$ can be expressed as

$$
\mathbf{H}_{\mathrm{t}}=\mathbf{H}_{\mathrm{S}}+\mathbf{H}_{\mathrm{B}}+\mathbf{H}_{\mathrm{SB}}
$$

where $\mathbf{H}_{\mathrm{S}}=\sum_{\mu} E_{\mu}|\mu\rangle\langle\mu|$ represents the Hamiltonian of system with eigen-state $|\mu\rangle$ and eigen-energy $E_{\mu}, \mathbf{H}_{\mathrm{B}}=$ $\sum_{n, k} \omega_{k} a_{n k}^{\dagger} a_{n k}$ corresponds to the environment, and $\mathbf{H}_{\mathrm{SB}}=$ $\sum_{n, k} g_{k}|n\rangle\langle n|\left(a_{n k}^{\dagger}+a_{n k}\right)$ describes the interaction between the system and environment. For the reduced density matrix, $\rho_{\mathrm{S}}(t)$ can be expressed as $\rho_{\mathrm{S}}(t)=\operatorname{Tr}_{\mathrm{B}}\left[\rho_{\mathrm{t}}(t)\right]$, where $\rho_{\mathrm{t}}(t)$ is the density matrix of the total system.

By treating $\mathbf{H}_{\mathrm{S}}$ exactly and $\mathbf{H}_{\mathrm{SB}}$ to the second order, we can derive a time-local master equation for the open system's density matrix $\rho_{\mathrm{S}}(t)$ as

$$
\frac{\mathrm{d}}{\mathrm{d} t} \rho_{\mathrm{S}}^{\mu v}(t)=-\mathrm{i} \omega_{\mu v} \rho_{\mathrm{S}}^{\mu v}+\sum_{\mu^{\prime} v^{\prime}} R_{\mu v, \mu^{\prime} v^{\prime}} \rho_{\mathrm{S}}^{\mu^{\prime} v^{\prime}},
$$

where $\omega_{\mu v}=\left(E_{\mu}-E_{v}\right) / \hbar$ is the transition frequency from the eigen-state $|\mu\rangle$ to $|v\rangle, R_{\mu v, \mu^{\prime} v^{\prime}}$ is the transfer rate from $\rho_{S}^{\mu^{\prime} v^{\prime}}$ to $\rho_{\mathrm{S}}^{\mu \nu}$. It is also called the Redfield tensor,

$$
\begin{aligned}
R_{\mu v, \mu^{\prime} v^{\prime}}= & \Gamma_{v^{\prime} v, \mu \mu^{\prime}}+\Gamma_{\mu^{\prime} \mu, v v^{\prime}}^{*}-\delta_{v^{\prime} v} \sum_{\kappa} \Gamma_{\mu \kappa, \kappa \mu^{\prime}} \\
& -\delta_{\mu^{\prime} \mu} \sum_{\kappa} \Gamma_{v \kappa, \kappa v^{\prime}}^{*}
\end{aligned}
$$

where the damping matrix is

$$
\Gamma_{\mu v, \mu^{\prime} v^{\prime}}=\frac{1}{\hbar^{2}} \sum_{m, n}\left\langle\mu\left|V_{m}\right| v\right\rangle\left\langle\mu^{\prime}\left|V_{n}\right| v^{\prime}\right\rangle C_{m n}\left(\omega_{v^{\prime} \mu^{\prime}}\right),
$$

$C_{m n}\left(\omega_{v^{\prime} \mu^{\prime}}\right)$ is the Fourier transform of bath correlation function

$$
C_{m n}(t)=\frac{\hbar}{\pi} \int_{-\infty}^{\infty} \mathrm{d} \omega J_{m n}(\omega)\left(n_{\mathrm{BE}}+1\right) \mathrm{e}^{-\mathrm{i} \omega t}
$$

with $n_{\mathrm{BE}}(\omega)=[\exp (\beta \hbar \omega)-1]^{-1}$ the Bose-Einstein distribution function and $J_{m n}(\omega)$ the spectral density. Here $\beta$ is inversely proportional to temperature.

Redfield theory is traditionally used in calculating the full quantum dynamics of the system's density matrix. Since it treats the system-bath couplings perturbatively, it is only valid when the intra-system couplings are much stronger than the system-bath couplings. Because the photosynthesis is in the intermediate regime, other approaches should be explored to describe the quantum dynamics of EET in photosynthesis [46, 63].

\section{B. Hierarchical Equation of Motion}

The HEOM formalism has become an important tool for studying dynamics of open quantum systems [64-67]. And it can be used to study the dynamics in different fields, such as chemistry [68], biology [69] and physics [70-73]. The establishment of the HEOM starts from the influence of the functional path integral [74-78], and then it is realized by using the path integration algorithm [79-81] and decomposing the environmental correlation functions appropriately [73, 80-84]. The HEOM couples the system's reduced density operator and a series of auxiliary density operators [79-81].

In the following, we describe in detail the theoretical approach of HEOM for photosynthetic EET [63, 85]. For a photosynthetic complex containing $N$ pigments, we can study its EET dynamics by using the Frenkel exciton Hamiltonian [44, 86, 87]

$$
H_{\mathrm{tot}}=H^{\mathrm{el}}+H^{\mathrm{ph}}+H^{\mathrm{el}-\mathrm{ph}}
$$

where

$$
\begin{aligned}
H^{\mathrm{el}} & =\sum_{j=1}^{N}|j\rangle \varepsilon_{j}\left\langle j\left|+\sum_{j \neq k}\right| j\right\rangle J_{j k}\langle k|, \\
H^{\mathrm{ph}} & =\sum_{j=1}^{N} H_{j}^{\mathrm{ph}}, \\
H^{\mathrm{el}-\mathrm{ph}} & =\sum_{j=1}^{N} H_{j}^{\mathrm{el}-\mathrm{ph}}=\sum_{j=1}^{N} V_{j} u_{j} .
\end{aligned}
$$

In the above, $|j\rangle$ represents the state where only the $j$ th pigment is in its excited state and all others are in their ground state, i.e., $|j\rangle=\left|\varphi_{1 g}\right\rangle\left|\varphi_{2 g}\right\rangle \cdots\left|\varphi_{j e}\right\rangle \cdots\left|\varphi_{N g}\right\rangle . \quad \varepsilon_{j}=\varepsilon_{j}^{0}+\lambda_{j}$ is the site energy of the $j$ th pigment, where $\varepsilon_{j}^{0}$ is the excitedstate energy of the $j$ th site in the absence of phonons and $\lambda_{j}$ is the reorganization energy of the $j$ th site. $J_{j k}$ is the electronic coupling between the $j$ th and $k$ th pigments. In Eq. (34), $H_{j}^{\mathrm{ph}}=\sum_{\xi} \omega_{\xi}\left(p_{\xi}^{2}+q_{\xi}^{2}\right) / 2$ is the Hamiltonian of the environmental phonons associated with the $j$ th pigment, with $q_{\xi}$ and $p_{\xi}$ dimensionless coordinate and conjugate momentum of the $\xi$ th mode. Eq. (35) is the coupling Hamiltonian between the $j$ th site and the phonon modes, with $V_{j}=|j\rangle\langle j|$ and $u_{j}=-\sum_{\xi} c_{j \xi} q_{\xi}$, where $c_{j \xi}$ being the coupling constant between the $j$ th pigment and $\xi$ th phonon mode.

The EET dynamics is given by the reduced density matrix

$$
\rho^{\mathrm{el}}(t)=\operatorname{Tr}_{\mathrm{ph}}\left\{\rho^{\mathrm{tot}}(t)\right\},
$$

where $\rho^{\text {tot }}(t)$ denotes the density matrix for the total system. In the interaction picture with respect to $H_{0}=H^{\mathrm{el}}+H^{\mathrm{ph}}$, the time evolution of the total density matrix $\rho_{\mathrm{I}}^{\text {tot }}(t)$ is governed by the Liouville equation,

$$
\partial_{t} \rho_{\mathrm{I}}^{\text {tot }}(t)=-\mathrm{i} \mathscr{L}_{\mathrm{el}-\mathrm{ph}}(t) \rho_{\mathrm{I}}^{\mathrm{tot}}(t),
$$

where $\mathscr{L}_{\mathrm{el}-\mathrm{ph}}(t)$ is the Liouville operator corresponding to the $H^{\mathrm{el}-\mathrm{ph}}$, which satisfies $\mathscr{L}_{\mathrm{el}-\mathrm{ph}}(t) \rho_{\mathrm{I}}^{\text {tot }}=$ $\left[\mathbf{H}^{\mathrm{e}-\mathrm{ph}}(t), \rho_{\mathrm{I}}^{\mathrm{tot}}\right]$. And $H^{\mathrm{el}-\mathrm{ph}}(t)=V_{j}(t) u_{j}(t)$, with $V_{j}(t)=$ $\exp \left(\mathrm{i} H^{\mathrm{el}} t\right) V_{j} \exp \left(-\mathrm{i} H^{\mathrm{el}} t\right), u_{j}(t)=\exp \left(\mathrm{i} H^{\mathrm{ph}} t\right) u_{j} \exp \left(-\mathrm{i} H^{\mathrm{ph}} t\right)$.

We suppose that the system and its environment is uncorrelated at the initial time [47], i.e., $\rho_{\mathrm{I}}^{\text {tot }}(0)=\rho_{\mathrm{I}}^{\mathrm{el}}(0) \rho_{\mathrm{eq}}^{\mathrm{ph}}$ with $\rho_{\mathrm{eq}}^{\mathrm{ph}}=\exp \left(-\beta H^{\mathrm{ph}}\right) / \operatorname{Tr}_{\mathrm{ph}} \exp \left(-\beta \mathrm{H}^{\mathrm{ph}}\right)$ and $\beta=1 / k_{\mathrm{B}} T$. 
By calculating the time integral of Eq. (37), the formal solution of reduced density matrix can be obtained as

$$
\rho_{\mathrm{I}}^{\mathrm{el}}(t)=\operatorname{Tr}_{\mathrm{ph}}\left\{\Gamma_{+} \exp \left[-\mathrm{i} \int_{0}^{\mathrm{t}} \mathrm{d} \tau \mathscr{L}_{\mathrm{el}-\mathrm{ph}}(\tau)\right] \rho_{\mathrm{eq}}^{\mathrm{ph}}\right\} \rho_{\mathrm{I}}^{\mathrm{el}}(0)
$$

where $\Gamma_{+}$is the forward time-ordering operator. The average over the linear bath operator $u_{j}(t)$ results in $\operatorname{Tr}_{\mathrm{ph}}\left\{u_{j}(t) \rho_{\mathrm{eq}}^{\mathrm{ph}}\right\}=$ 0 and $\operatorname{Tr}_{\mathrm{ph}}\left\{u_{j}(t) u_{j}(0) \rho_{\mathrm{eq}}^{\mathrm{ph}}\right\} \equiv C(t) \equiv C_{\text {real }}(t)+\mathrm{i} C_{\text {imag }}(t)$, where $C_{\text {real }}(t)$ and $C_{\text {imag }}(t)$ are the real and imaginary parts of the correlation function of bath, respectively. Consequently, Eq. (38) is simplified to be

$$
\rho_{\mathrm{I}}^{\mathrm{el}}(t)=U(t) \rho_{\mathrm{I}}^{\mathrm{el}}(0)
$$

with the time propagator of the reduced density matrix

$$
U(t)=\Gamma_{+} \exp \left[-\int_{0}^{t} W(\tau) \mathrm{d} \tau\right]
$$

where $W(t)=\int_{0}^{t} \mathrm{~d} \tau \mathscr{L}_{\mathrm{z}}(t) C_{\text {real }}(t-\tau) \mathscr{L}_{\mathrm{z}}(\tau)+\mathrm{i} \mathscr{L}_{\mathrm{z}}(t) C_{\text {imag }}(t-$ $\tau) \mathscr{S}_{\mathrm{z}}(\tau), \mathscr{L}_{\mathrm{z}}(t)=\left[V_{j}(t), \ldots\right], \mathscr{S}_{\mathrm{z}}(t)=\left[V_{j}(t), \ldots\right]_{+}$.

The coupling of the $j$ th pigment to the environmental phonons can be specified by the spectral density $J_{j}(\omega)$. We further assume the spectral density as the overdampedBrownian oscillator model, $J_{j}(\omega)=2 \lambda_{j} \gamma_{j} \omega /\left(\omega^{2}+\gamma_{j}^{2}\right)$ with $\gamma_{j}^{-1}$ being the relaxation time and $\lambda_{j}$ the reorganization energy [88].

Thus, the bath correlation function can be calculated as

$$
C(t)=C_{\text {real }}-\mathrm{i} C_{\text {imag }}=\int_{-\infty}^{\infty} \frac{\mathrm{d} \omega}{\pi} J(\omega) \frac{\mathrm{e}^{\mathrm{i} \omega t}}{\mathrm{e}^{\beta \omega-1}},
$$

where

$$
\begin{aligned}
C_{\text {real }}(t) & =\int_{-\infty}^{\infty} \frac{\mathrm{d} \omega}{\pi} J(\omega) \operatorname{coth} \frac{\beta \omega}{2} \cos \omega t, \\
C_{\text {imag }}(t) & =-\int_{-\infty}^{\infty} \frac{\mathrm{d} \omega}{\pi} J(\omega) \sin \omega t
\end{aligned}
$$

By unitary transformation, we can obtain the reduced density matrix $\rho^{\mathrm{el}}(t)$ in the Schrödinger picture as $\rho^{\mathrm{el}}(t)=$ $U_{\mathrm{el}}(t) \rho_{\mathrm{I}}^{\mathrm{el}}(t) U_{\mathrm{el}}^{\dagger}(t)=\exp \left(-\mathrm{i} H^{\mathrm{el}} t\right) \rho_{\mathrm{I}}^{\mathrm{el}}(t) \exp \left(\mathrm{i} H^{\mathrm{el}} t\right)$. And its time derivative can be expressed as

$$
\partial_{t} \rho^{\mathrm{el}}(t)=-\mathrm{i} \mathscr{L}_{\mathrm{S}} \rho^{\mathrm{el}}(t)-\mathrm{i} \mathscr{L}_{\mathrm{z}} \sigma_{1}(t)
$$

where $\mathscr{L}_{\mathrm{S}}=\left[H^{\mathrm{el}}, \ldots\right]$ is the commutator of the system Hamiltonian. Here $\sigma_{1}(t)$ is an auxiliary operator, which is

$$
\begin{aligned}
\sigma_{1}(t)= & \Gamma_{+}\left\{\int_{0}^{t} \mathrm{~d} \tau\left[-\mathrm{i} C_{\text {real }}(t-\tau) \mathscr{L}_{\mathrm{z}}(\tau)+C_{\text {imag }}(t-\tau) S_{\mathrm{Z}}(\tau)\right]\right. \\
& \left.U_{\mathrm{el}}(t) U(t)\right\} \rho^{\mathrm{el}}(0) .
\end{aligned}
$$

Under the high-temperature condition, i.e., $\beta \hbar \gamma_{j}<1$, $\mathrm{e}^{\beta \omega}-$ $1 \approx \beta \omega$, we can obtain

$$
C_{\text {real }}(t)=\frac{2 \lambda_{j}}{\beta} \mathrm{e}^{-\gamma_{j} t}, C_{\text {imag }}(t)=-2 \lambda_{j} \gamma_{j} \mathrm{e}^{-\gamma_{j} t} .
$$

Thus, an arbitrary $n$ th-order auxiliary operator can be established as

$$
\begin{aligned}
\sigma_{\mathbf{n}}(t)= & \Gamma_{+}\left\{\int_{0}^{t} \mathrm{~d} \tau_{1} \Theta_{n_{1}}\left(\tau_{1}\right) \mathrm{e}^{-\gamma_{j}\left(t-\tau_{1}\right)} \int_{0}^{t} \mathrm{~d} \tau_{2} \Theta_{n_{2}}\left(\tau_{2}\right) \mathrm{e}^{-\gamma_{j}\left(t-\tau_{2}\right)}\right. \\
& \left.\ldots \int_{0}^{t} \mathrm{~d} \tau_{N} \Theta_{n_{N}}\left(\tau_{N}\right) \mathrm{e}^{-\gamma_{j}\left(t-\tau_{N}\right)} U_{\mathrm{el}}(t) U(t)\right\} \rho^{\mathrm{el}}(0) \\
= & \Gamma_{+}\left\{\sum_{j=1}^{N}\left[\int_{0}^{t} \mathrm{~d} \tau \Theta_{n_{j}}(\tau) \mathrm{e}^{-\gamma_{j}(t-\tau)}\right]^{n_{j}}\right. \\
& \left.U_{\mathrm{el}}(t) U(t)\right\} \rho^{\mathrm{el}}(0),
\end{aligned}
$$

where $\Theta_{n_{j}}(\tau)=-\mathrm{i} \frac{2 \lambda_{j}}{\beta} \mathscr{L}_{\mathrm{Z}}(\tau)-2 \lambda_{j} \gamma_{j} S_{\mathrm{Z}}(\tau)$. Then, we apply the time derivative on both sides of Eq. (47) to derive the equation of motion for the $n$ th-order auxiliary operator as

$$
\begin{aligned}
\partial_{t} \sigma_{\mathbf{n}}(t)= & -\mathrm{i} \mathscr{L}_{\mathrm{S}} \sigma_{\mathbf{n}}(t)+\sum_{j=1}^{N} n_{j}\left(-\gamma_{j}\right) \sigma_{\mathbf{n}}(t)+\sum_{j=1}^{N} n_{j} \Theta_{n_{j}}(\tau) \sigma_{\mathbf{n}_{j-}}(t) \\
& +\sum_{j=1}^{N}(-\mathrm{i}) \mathscr{L}_{\mathrm{z}} \sigma_{\mathbf{n}_{j+}}(t)
\end{aligned}
$$

where $\mathbf{n}=\left(n_{1}, \ldots, n_{j}, \ldots, n_{N}\right), \mathbf{n}_{j \pm}=\left(n_{1}, \ldots, n_{j} \pm 1, \ldots, n_{N}\right)$ are three sets of nonnegative integers.

Since the hierarchically-coupled Eq. (48) continues to infinity, we terminate Eq. (48) at a finite stage as

$$
\partial_{t} \sigma_{\mathbf{n}}(t)=-\mathrm{i} \mathscr{L}_{\mathrm{S}} \sigma_{\mathbf{n}}(t)
$$

for the integers $\mathbf{n}=\left(n_{1}, n_{2}, \ldots, n_{N}\right)$ satisfying

$$
\mathscr{N}=\sum_{j=1}^{N} n_{j} \gg \frac{\omega_{e}}{\min \left(\gamma_{1}, \gamma_{2}, \cdots, \gamma_{N}\right)},
$$

where $\omega_{e}$ is a characteristic frequency for $\mathscr{L}_{\mathrm{S}}$. The number of the operators $\{\sigma(\mathbf{n}, t)\}$ is $(N+\mathscr{N}) ! /(N ! \mathscr{N} !)$. Note that Eq. (48) is not suitable for the condition of low-temperature [85].

Above all, we only analyze the type of Debye spectral density. It has been proved that HEOM is suitable for any spectral density. Several different methods have been proposed to decompose any spectral density into analytical forms suitable for HEOM processing [65]. The photosynthetic complex is essentially an open quantum system. Therefore, HEOM can be applied not only to the EET of photosynthesis but also to the related problems in open quantum systems, such as the dynamics of the dissipative electron systems [81], the nonMarkovian entanglement dynamics [89] and so on.

As demonstrated in Ref. [63], Eq. (48) can describe not only quantum coherent wave-like motion in the Redfield regime, but also incoherent hopping in the Förster regime and an intermediate EET regime in a unified manner. However, although the HEOM numerically exactly produces the quantum dynamics in all three regimes, it takes intolerable computation time, which is exponential in the system's size and the number of exponents in the bath correlation function [88]. Recently, by sophisticated mapping, we experimentally accelerate the exact simulation of the HEOM in the NMR [88]. 


\section{Quantum path integral}

In this subsection, we use a general model to illustrate how path integral can be used in an open quantum system [90-92]. Consider a general quantum system in a bath environment, and assume that the bath is a regular ensemble. The Hamiltonian of the whole system can be expressed as

$$
H_{\mathrm{tot}}=H_{\mathrm{S}}(t)+H_{\mathrm{B}}-\sum_{a} Q_{a} F_{a}
$$

Here, $H_{\mathrm{S}}(t)$ is the Hamiltonian of the system. $H_{\mathrm{B}}$ is the Hamiltonian of the bath. The last term of Eq. 51) describes the coupling of system and bath, formally decomposed into several dissipative modes, where $Q_{a}$ is the operator of system, and $F_{a}$ is the bath's operator.

The system's reduced density matrix $\rho(t)$ can be defined as

$$
\rho(t)=\operatorname{Tr}_{B} \rho_{\text {tot }}(t)=\mathscr{U}\left(t, t_{0}\right) \rho\left(t_{0}\right) .
$$

Here, $\mathscr{U}\left(t, t_{0}\right)$ is the propagator. And Eq. (52) is given at the operator level. However, the expression of path integral must be expanded under certain representation. Consider $|\alpha\rangle$ to be a set of system's complete bases. Under $\alpha$ representation, assuming that $\boldsymbol{\alpha}=\left(\alpha, \alpha^{\prime}\right)$, Eq. (52) can be expressed as ()

$$
\rho(\boldsymbol{\alpha}, t)=\int \mathrm{d} \boldsymbol{\alpha}_{0} \mathscr{U}\left(\boldsymbol{\alpha}, t ; \boldsymbol{\alpha}_{0}, t_{0}\right) \rho\left(\boldsymbol{\alpha}_{0}, t_{0}\right)
$$

where $\mathscr{U}\left(\boldsymbol{\alpha}, t ; \boldsymbol{\alpha}_{0}, t_{0}\right)$ reads [74]

$$
\mathscr{U}\left(\boldsymbol{\alpha}, t ; \boldsymbol{\alpha}_{0}, t_{0}\right)=\int_{\alpha_{0}\left[t_{0}\right]}^{\alpha[t]} \mathrm{D} \boldsymbol{\alpha} \mathrm{e}^{\mathrm{i} S[\alpha]} F[\alpha] \mathrm{e}^{-\mathrm{i} S\left[\alpha^{\prime}\right]} .
$$

Here, $F$ is the influence functional, which mainly expresses the effect of interaction between the system and bath. $S[\alpha]$ is the classical action functional along a path $\alpha(\tau)$ between starting point $\alpha\left(t_{0}\right)=\boldsymbol{\alpha}_{0}$ and ending point $\alpha(t)=\boldsymbol{\alpha}$. Note that the two endpoints of this path are fixed. If the systembath interaction is not considered, i.e., $F=1$, the dynamics of the system is a completely-coherent process, that is to say, when $F=1$, we have $\partial_{t} \mathscr{U}=-i \mathscr{L} \mathscr{U}$, where $\mathscr{L}$ is the Liouville operator of the system, i.e., $\mathscr{L}=\left[H_{\mathrm{S}}(t), \ldots\right]$.

Now, the key quantity we consider is the influence functional $F$ induced by the system-bath interaction. Let $\boldsymbol{a}=\left(a a^{\prime}\right)$ denote a pair of dissipative modes, and introduce the definition [79]

$$
\tilde{\mathscr{Q}}(t ; \boldsymbol{\alpha})=\tilde{Q}_{a a^{\prime}}(t ; \alpha)-\tilde{Q}_{a a^{\prime}}^{\prime}\left(t ; \alpha^{\prime}\right)
$$

where

$$
\begin{aligned}
& \tilde{Q}_{a}(t ; \alpha)=\tilde{Q}_{a a^{\prime}}=\int_{t_{0}}^{t} \mathrm{~d} \tau C_{\boldsymbol{a}}(t-\tau) Q_{a^{\prime}}[\alpha(\tau)], \\
& \tilde{Q}_{\boldsymbol{a}}^{\prime}(t ; \alpha)=\int_{t_{0}}^{t} \mathrm{~d} \tau C_{\boldsymbol{a}}^{*}(t-\tau) Q_{a^{\prime}}\left[\alpha^{\prime}(\tau)\right], \\
& \mathscr{Q}_{a}[\boldsymbol{\alpha}(t)]=Q_{a}[\alpha(t)]-Q_{a}\left[\alpha^{\prime}(t)\right] .
\end{aligned}
$$

Here, for any operator $\hat{O}, \mathscr{Q} \hat{O}=\left[Q_{a}, \hat{O}\right] . C_{a}(t-\tau)=C_{a a^{\prime}}(t-$ $\tau)=\left\langle\hat{F}_{a}(t) \hat{F}_{a^{\prime}}(\tau)\right\rangle_{B}$ is the correlation function of the bath.
The final expression of influence functional $F$ reads [79]

$$
F[\boldsymbol{\alpha}]=\exp \left\{-\int_{t_{0}}^{t} \mathrm{~d} \tau R[\tau ; \boldsymbol{\alpha}]\right\}
$$

where

$$
R[\tau ; \boldsymbol{\alpha}]=\sum_{\boldsymbol{\alpha}} \mathscr{Q}[\boldsymbol{\alpha}(t)] \tilde{\mathscr{Q}}_{a}(t ; \boldsymbol{\alpha})
$$

As a powerful formulism, path integral can be used to calculate quantum dynamics under semi-classical approximation. When dealing with many-body quantum dynamical problems, path integral does not introduce uncontrolled approximation. However, in general, the solution of path integral is not easy, because it should discretize the paths based on time slices in order to generate multi-dimensional integrals. Therefore, in the long-time limit, the dimension of the integral may be very high. In addition, numerical techniques for evaluating path integrals in real time and imaginary time are different.

\section{Coherent Modified Redfield theory}

We have introduced the MRT in Sec. C. In this section, we derive the CMRT by generalizing the MRT. Although the CMRT approach adopts the same basis of the MRT, it can deal with coherent evolution of excitonic systems [93, 94].

In this section, we adopt the same model as Sec. C. In $\mathrm{Sec}$. C, we have discussed that the main idea of MRT is to divide the Hamiltonian into a zeroth-order Hamiltonian including the diagonal system-bath couplings in the exciton basis,

$$
H_{0}=H^{\mathrm{el}}+H^{\mathrm{ph}}+\sum_{\alpha}|\alpha\rangle\langle\alpha| \cdot\left(H^{\mathrm{el}-\mathrm{ph}}\right)_{\alpha \alpha},
$$

and the off-diagonal system-bath couplings in the exciton basis as a perturbation,

$$
V=\sum_{\alpha \neq \beta}|\alpha\rangle\langle\beta| \cdot\left(H^{\mathrm{el}-\mathrm{ph}}\right)_{\alpha \beta} .
$$

Following Born approximation and second-order truncation [95], we derive the master equation for coherent EET dynamics,

$$
\begin{aligned}
& \dot{\sigma}_{\alpha \beta}(t)=-\mathrm{i}\left(\varepsilon_{\alpha}-\varepsilon_{\beta}\right) \sigma_{\alpha \beta}(t) \\
& +\sum_{f}\left[R_{\alpha f}(t) \sigma_{f f}(t)-R_{f \alpha}(t) \sigma_{\alpha \alpha}(t)\right] \cdot \delta_{\alpha \beta} \\
& -\left[R_{\alpha \beta}^{p d}(t)+\frac{1}{2} \sum_{f}\left(R_{f \alpha}(t)+R_{f \beta}(t)\right)\right] \sigma_{\alpha \beta}(t),
\end{aligned}
$$

where

$$
\begin{aligned}
R_{\alpha \beta}(t) & =2 \operatorname{Re} \int_{0}^{t} \mathrm{~d} \tau F_{\beta}^{*}(\tau) A_{\alpha}(\tau) X_{\alpha \beta}(\tau), \\
A_{\alpha}(t) & =\mathrm{e}^{-\mathrm{i} \varepsilon_{\alpha} t-g_{\alpha \alpha \alpha \alpha}(t)} \\
F_{\alpha}(t) & =\mathrm{e}^{-\mathrm{i}\left(\varepsilon_{\alpha}-2 \lambda_{\alpha \alpha \alpha \alpha}\right) t-g_{\alpha \alpha \alpha \alpha}^{*}(t)},
\end{aligned}
$$




$$
\begin{aligned}
X_{\alpha \beta}(t)= & \mathrm{e}^{2\left[g_{\alpha \alpha \beta \beta}(t)+\mathrm{i} \lambda_{\alpha \alpha \beta \beta} t\right]} \\
& \times\left[\ddot{g}_{\beta \alpha \alpha \beta}(t)-\left(\dot{g}_{\beta \alpha \alpha \alpha}(t)-\dot{g}_{\beta \alpha \beta \beta}(t)-2 \mathrm{i} \lambda_{\beta \alpha \beta \beta}\right)\right. \\
& \left.\times\left(\dot{g}_{\alpha \beta \alpha \alpha}(t)-\dot{g}_{\alpha \beta \beta \beta}(t)-2 i \lambda_{\alpha \beta \beta \beta}\right)\right] \\
g_{\alpha \beta \gamma \delta}(t)= & \sum_{n, m} c_{n}^{\alpha} c_{n}^{\beta *} c_{m}^{\gamma} c_{m}^{\delta *} g_{n m}(t), \\
\lambda_{\alpha \beta \gamma \delta}= & \sum_{n} c_{n}^{\alpha} c_{n}^{\beta *} c_{n}^{\gamma} c_{n}^{\delta *} \lambda_{n} .
\end{aligned}
$$

Here the lineshape function is

$$
\begin{aligned}
g_{n m}(t)= & \int_{0}^{\infty} \mathrm{d} \omega \frac{J_{n m}(\omega)}{\omega^{2}}\left\{\operatorname{coth}\left(\frac{\omega}{2 \mathrm{k}_{\mathrm{B}} \mathrm{T}}\right)[1-\cos (\omega \mathrm{t})]\right. \\
& +\mathrm{i}[\sin (\omega t)-\omega t]\}
\end{aligned}
$$

and the spectral density is

$$
J_{n m}(\omega)=\sum_{i} g_{n i} g_{m i} \omega_{i}^{2} \delta\left(\omega-\omega_{i}\right)
$$

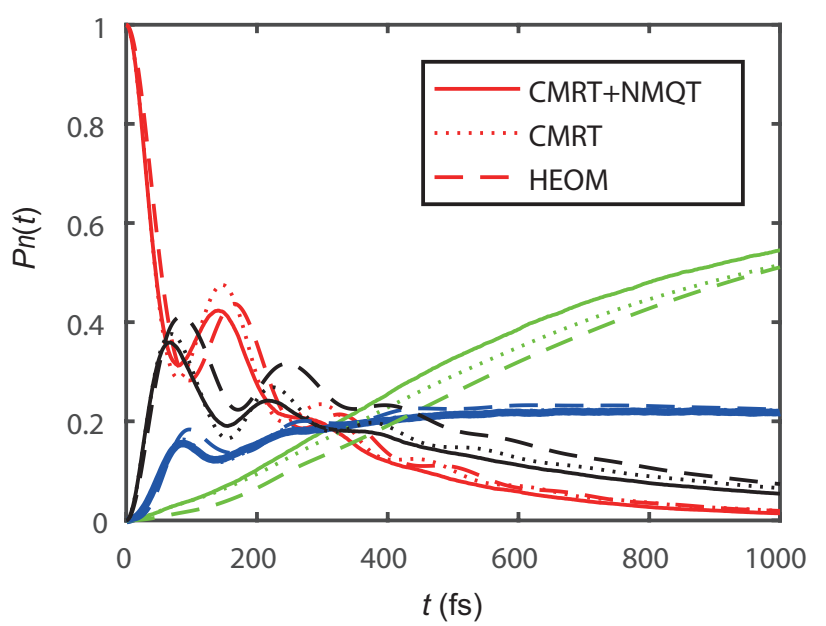

FIG. 2: (Color online) $P_{n}(t)$ vs $t$ of FMO with $P_{n}(0)=\delta_{n 6}$ [96]. The simulations are performed with $T=77 \mathrm{~K}, \lambda=35 \mathrm{~cm}^{-1}$, and the effective Hamiltonian from Ref. [46]. Solid curves are obtained by the combined Lindblad-form-CMRT-and-NMQJ approach,dotted lines are calculated by the original CMRT, and dashed curves are results by the HEOM. Red curves are for site 6 , green curves for site 3 , blue curves for site 4 and black curves for site 5. For the sake of clarity, other sites' population dynamics are small and thus not shown here.

In Fig. 2, we compare the population dynamics by the original CMRT, the HEOM, and the combined approach of Lindblad-form CMRT and NMQT [50, 96]. It is shown that the CMRT captures both the coherent oscillations at the shorttime regime and the incoherent EET dynamics at the longtime regime. We notice that for sites 6 and 3, the difference between the prediction by the HEOM and the CMRT may be more significant. It was remarked that when the energy gap is small and the exciton-phonon coupling is strong, the CMRT neglects the dynamical localization. Furthermore, the CMRT-NMQT approach underestimates the coherent evolution, because it might be attributed to the recast the CMRT into the Lindblad form in order to utilize the NMQT approach [50, 96].

By comparison, we can find that the master equation (63) has the similar form as the traditional Redfield equation (28). Because it is time-local, it is easy to propagate and applicable to large multichromophoric systems. The excitonic Hamiltonian $H^{\mathrm{el}}$ results in the coherent dynamics as described by the first term in Eq. (63). The off-diagonal exciton-phonon couplings in the exciton basis $V$ lead to the incoherent population transfer, cf. the second term therein. The last term stands for the dephasing processes induced by both the pure-dephasing and the dissipation effects. The CMRT is non-Markovian since we have not invoked the Markovian approximation in deriving Eq. (63). Moreover, as in Ref. [56], we would obatain the same absorption spectrum with lifetime broadening effects due to the dephasing terms in Eq. (63). The CMRT is applicable to some problems beyond the original MRT, such as time-resolved spectroscopy and control problems [96].

\section{E. Small-Polaron quantum master equation}

In addition to the methods mentioned above, a new theory has recently been developed, which interpolates between the Förster and Redfield theories by using small-polaron transformation [97]. This theory is based on the second-order perturbative truncation of the renormalized electron-phonon coupling.

We consider a model consisting of a donor (D) and an acceptor (A) [97]. And we use $|\mathrm{g}\rangle$ to represent the ground state of the $\mathrm{D}$ and $\mathrm{A},|\mathrm{D}\rangle(|\mathrm{A}\rangle)$ to represent that only $\mathrm{D}(\mathrm{A})$ is in the excited state. In this subsection, we only consider the situation when there is only single electronic-excitation.

Initially, we assume that the state of the system is in the ground state $|\mathrm{g}\rangle$ and the bath is in thermal equilibrium. Then, a laser pulse selectively excites $|\mathrm{g}\rangle$ to $|\mathrm{D}\rangle$. The EET dynamics for $t>0$ can be described by the total Hamiltonian

$$
H=H_{\mathrm{s}}+H_{\mathrm{sb}}+H_{\mathrm{b}},
$$

where $H_{\mathrm{s}}=H_{\mathrm{s}}^{\mathrm{p}}+H_{\mathrm{s}}^{\mathrm{c}}$ represents the system Hamiltonian with $H_{\mathrm{s}}^{\mathrm{p}}=E_{\mathrm{D}}|\mathrm{D}\rangle\left\langle\mathrm{D}\left|+E_{\mathrm{A}}\right| \mathrm{A}\right\rangle\langle\mathrm{A}|$ and $H_{\mathrm{s}}^{\mathrm{c}}=J(|\mathrm{D}\rangle\langle\mathrm{A}|+| \mathrm{A}\rangle\langle\mathrm{D}|), J$ is the coupling between $|\mathrm{D}\rangle$ and $|\mathrm{A}\rangle, E_{\mathrm{D}}\left(E_{\mathrm{A}}\right)$ is the energy of state $|\mathrm{D}\rangle(|A\rangle)$ relative to ground state $|\mathrm{g}\rangle, H_{\mathrm{b}}$ is the Hamiltonian of the bath, $H_{\mathrm{sb}}=B_{\mathrm{D}}|\mathrm{D}\rangle\left\langle\mathrm{D}\left|+B_{\mathrm{A}}\right| \mathrm{A}\right\rangle\langle\mathrm{A}|$ is the systembath interaction Hamiltonian, with $B_{\mathrm{D}}\left(B_{\mathrm{A}}\right)$ the bath operator coupled to $|\mathrm{D}\rangle(|\mathrm{A}\rangle)$. Here, we assume a spin-boson-type model,

$$
\begin{aligned}
& H_{\mathrm{b}}=\sum_{n} \hbar \omega_{n}\left(b_{n}^{\dagger} b_{n}+\frac{1}{2}\right), \\
& B_{\mathrm{D}}=\sum_{n} \hbar \omega_{n} g_{n D}\left(b_{n}^{\dagger}+b_{n}\right), \\
& B_{\mathrm{A}}=\sum_{n} \hbar \omega_{n} g_{n A}\left(b_{n}^{\dagger}+b_{n}\right),
\end{aligned}
$$

where $b_{n}^{\dagger}\left(b_{n}\right)$ is the creation (annihilation) operator of the $n$th mode, and its corresponding frequency $\omega_{n}$. According to the initial condition, $\rho(0)=\sigma(0) \mathrm{e}^{-\beta H_{\mathrm{b}}} / Z$, where $\beta=1 / k_{\mathrm{B}} T$, 
$Z=\operatorname{Tr}_{\mathrm{b}}\left\{\mathrm{e}^{-\beta H_{\mathrm{b}}}\right\}$, and $\sigma=|\mathrm{D}\rangle\langle\mathrm{D}|$. The Liouville operator corresponding to the above Hamiltonians can be expressed as $\mathscr{L}$, $\mathscr{L}_{\mathrm{s}}^{p}, \mathscr{L}_{\mathrm{s}}^{c}, \mathscr{L}_{\mathrm{sb}}$, and $\mathscr{L}_{\mathrm{b}}$. The evolution of the density operator over time reads

$$
\frac{\mathrm{d} \rho(t)}{\mathrm{d} t}=-\mathrm{i} \mathscr{L} \rho(t)=-\mathrm{i}\left(\mathscr{L}_{\mathrm{s}}^{\mathrm{p}}+\mathscr{L}_{\mathrm{s}}^{\mathrm{c}}+\mathscr{L}_{\mathrm{sb}}+\mathscr{L}_{\mathrm{b}}\right) \rho(t) .
$$

As we have discussed in the above subsections, when the coupling to the bath is weak, the Redfield theory can be employed, while for the strong coupling to the bath, the Förster theory is applicable. The method developed below interpolates between these two limits by combining the polaron transformation [98-102] and a quantum master equation formulism up to the second order [45].

Applying the small-polaron transformation generated by $G=\sum_{n}\left(b_{n}^{\dagger}-b_{n}\right)\left(g_{\mathrm{nD}}|\mathrm{D}\rangle\left\langle\mathrm{D}\left|+g_{\mathrm{nA}}\right| \mathrm{A}\right\rangle\langle\mathrm{A}|\right)$ to Eq. (74), we can obtain the time evolution equation for $\tilde{\rho}(t)=\mathrm{e}^{G} \rho(t) \mathrm{e}^{-G}$ as

$$
\frac{\mathrm{d} \tilde{\rho}(t)}{\mathrm{d} t}=-\mathrm{i}\left(\tilde{\mathscr{L}}_{\mathrm{s}}^{\mathrm{p}}+\tilde{\mathscr{L}}_{\mathrm{s}}^{\mathrm{c}}+\mathscr{L}_{\mathrm{b}}\right) \tilde{\rho}(t),
$$

where $\tilde{\mathscr{L}}_{\mathrm{s}}^{\mathrm{p}}$ and $\tilde{\mathscr{L}}_{\mathrm{s}}^{\mathrm{c}}$ are the quantum Liouville operators for

$$
\begin{aligned}
\tilde{\mathbf{H}}_{\mathrm{s}}^{p} & =\tilde{E}_{\mathrm{D}}|\mathrm{D}\rangle\left\langle\mathrm{D}\left|+\tilde{E}_{\mathrm{A}}\right| \mathrm{A}\right\rangle\langle\mathrm{A}|, \\
\tilde{\mathbf{H}}_{\mathrm{s}}^{c} & =J\left(\theta_{\mathrm{D}}^{\dagger} \theta_{\mathrm{A}}|\mathrm{D}\rangle\left\langle\mathrm{A}\left|+\theta_{\mathrm{A}}^{\dagger} \theta_{\mathrm{D}}\right| \mathrm{A}\right\rangle\langle\mathrm{D}|\right) .
\end{aligned}
$$

In the above equations, $\tilde{E}_{\mathrm{D}}=E_{\mathrm{D}}-\sum_{n} g_{\mathrm{nD}}^{2} \hbar \omega_{n}$ and $\tilde{E}_{\mathrm{A}}=$ $E_{\mathrm{A}}-\sum_{n} g_{n A}^{2} \hbar \omega_{n}, \theta_{\mathrm{D}}=\exp \left\{-\sum_{n} g_{\mathrm{nD}}\left(b_{n}^{\dagger}-b_{n}\right)\right\}$ and $\theta_{\mathrm{A}}=$ $\exp \left\{-\sum_{n} g_{\mathrm{nA}}\left(b_{n}^{\dagger}-b_{n}\right)\right\}$, and $\theta_{\mathrm{D}}^{\dagger}$ and $\theta_{\mathrm{A}}^{\dagger}$ are their Hermitian conjugates. The initial condition transforms to $\tilde{\rho}(0)=\sigma(0) \theta_{\mathrm{D}}^{\dagger} \mathrm{e}^{-\beta H_{\mathrm{b}}} \theta_{\mathrm{D}} / Z$, which corresponds to the nonequilibrium state of the bath.

In order to derive the quantum master equation, the transformed total Hamiltonian can be divided as $\tilde{H}=\tilde{H}_{0}+\tilde{H}_{1}$. The zeroth-order term is

$$
\tilde{H}_{0}=\tilde{H}_{0, \mathrm{~s}}+H_{\mathrm{b}},
$$

where

$$
\begin{aligned}
\tilde{H}_{0, \mathrm{~s}} & =\tilde{E}_{\mathrm{D}}|\mathrm{D}\rangle\left\langle\mathrm{D}\left|+\tilde{E}_{\mathrm{A}}\right| \mathrm{A}\right\rangle\langle\mathrm{A}|+J \omega(|\mathrm{D}\rangle\langle\mathrm{A}|+| \mathrm{A}\rangle\langle\mathrm{D}|)(79) \\
\omega & =\left\langle\theta_{\mathrm{D}}^{\dagger} \theta_{\mathrm{A}}\right\rangle=\left\langle\theta_{\mathrm{A}}^{\dagger} \theta_{\mathrm{D}}\right\rangle=\mathrm{e}^{-\sum_{n} \operatorname{coth}\left(\beta \hbar \omega_{n} / 2\right) \delta g_{n}^{2} / 2}
\end{aligned}
$$

with $\delta g_{\mathrm{n}}=g_{\mathrm{nD}}-g_{\mathrm{nA}}$. The first-order term $\tilde{H}_{1}$ is defined as

$$
\tilde{H}_{1}=\tilde{H}_{\mathrm{s}}^{\mathrm{c}}-\left\langle\tilde{H}_{\mathrm{s}}^{\mathrm{c}}\right\rangle=J\left(\tilde{\mathrm{B}}|\mathrm{D}\rangle\left\langle\mathrm{A}\left|+\tilde{\mathrm{B}}^{\dagger}\right| \mathrm{A}\right\rangle\langle\mathrm{D}|\right),
$$

where $\tilde{\mathrm{B}}=\theta_{\mathrm{D}}^{\dagger} \theta_{\mathrm{A}}-\omega,\langle\tilde{\mathrm{B}}\rangle=\left\langle\tilde{\mathrm{B}}^{\dagger}\right\rangle=0$. We take $J \tilde{\mathrm{B}}$ and $J \tilde{\mathrm{B}}^{\dagger}$ as perturbations, which remain small in both limits of weak and strong system-bath couplings. Thus, the second-order quantum master equation with respect to $\tilde{H}_{1}$ is valid in both limits.

In the interaction picture with respect to $\tilde{H}_{0}$, the time evolution equation of density operator $\tilde{\rho}_{\mathrm{I}}(t)=\exp \left\{i \tilde{L}_{0} t\right\} \tilde{\rho}(t)$ is

$$
\frac{\mathrm{d}}{\mathrm{d} t} \tilde{\rho}_{\mathrm{I}}(t)=-\mathrm{i} \tilde{L}_{1, \mathrm{I}}(t) \tilde{\rho}_{\mathrm{I}}(t),
$$

where $\tilde{L}_{1, \mathrm{I}}(t)$ is the quantum Liouville operator corresponding to

$$
\tilde{H}_{1, \mathrm{I}}(t)=J\left[\tilde{\mathrm{B}}(t) T(t)+\tilde{B}^{\dagger}(t) T^{\dagger}(t)\right] .
$$

Here, $\quad \tilde{\mathrm{B}}(t)=\exp \left\{\mathrm{i} H_{\mathrm{b}} t / \hbar\right\} \tilde{B} \exp \left\{-\mathrm{i} H_{\mathrm{b}} t / \hbar\right\} \quad$ and $\quad T(t)=$ $\exp \left\{\mathrm{i} \tilde{H}_{0, s} t / \hbar\right\}|\mathrm{D}\rangle\langle\mathrm{A}| \exp \left\{-\mathrm{i} \tilde{H}_{0, \mathrm{~s}} t / \hbar\right\}$.

We apply the standard projection-operator technique [97] with $P(\cdot) \equiv \rho_{\mathrm{b}} \operatorname{Tr}_{\mathrm{b}}\{\cdot\}$ and $Q=1-P$ to Eq. (82), and then make second-order approximations with respect to $\mathscr{L}_{1, \mathrm{I}}(t)$ for both the homogeneous and inhomogeneous terms consistently to obtain

$$
\begin{aligned}
\frac{\mathrm{d}}{\mathrm{d} t} P \tilde{\rho}_{\mathrm{I}}(t)= & -\mathrm{i} P \tilde{\mathscr{L}}_{1, \mathrm{I}}(t) Q \tilde{\rho}(0)-\int_{0}^{t} \mathrm{~d} \tau P \tilde{\mathscr{L}}_{1, \mathrm{I}}(t) \tilde{\mathscr{L}}_{1, \mathrm{I}}(\tau) \\
& \times\left(Q \tilde{\rho}(t)+P \tilde{\rho}_{\mathrm{t}}(\tau)\right),
\end{aligned}
$$

where $\quad P \tilde{L}_{1, \mathrm{I}}(t) P=0 \quad$ and $\quad Q \tilde{\rho}(0)=\sigma(0) \times$ $\left(\theta_{\mathrm{D}}^{\dagger} \mathrm{e}^{-\beta H_{\mathrm{b}}} \theta_{\mathrm{D}}-\mathrm{e}^{-\beta H_{\mathrm{b}}}\right) / Z$ has been used. In the above equation, $P \tilde{\rho}_{\mathrm{I}}(\tau)$ can be replaced by $P \tilde{\rho}_{\mathrm{I}}(t)$ without affecting the accuracy up to the second order. By tracing the bath degrees of freedom to the resulting equation, we obtain the following time-local quantum master equation for $\tilde{\sigma}_{\mathrm{I}}(t)=\operatorname{Tr}_{\mathrm{b}}\left\{\tilde{\rho}_{\mathrm{I}}(t)\right\}$,

$$
\frac{\mathrm{d}}{\mathrm{d} t} \tilde{\sigma}_{\mathrm{I}}(t)=-\mathscr{R} \tilde{\sigma}_{\mathrm{I}}(t)+\mathscr{I}(t),
$$

where

$$
\begin{aligned}
\mathscr{R}(t)= & \int_{0}^{t} \mathrm{~d} \tau \operatorname{Tr}_{b}\left\{\tilde{\mathscr{L}}_{1, \mathrm{I}}(t) \mathscr{L}_{1, \mathrm{I}}(\tau) \rho_{\mathrm{b}}\right\}, \\
\mathscr{I}(t)= & -\operatorname{iTr}_{\mathrm{b}}\left\{\tilde{\mathscr{L}}_{1, \mathrm{I}}(t) Q \tilde{\rho}(0)\right\} \\
& -\int_{0}^{t} \mathrm{~d} \tau \operatorname{Tr}_{b}\left\{\tilde{\mathscr{L}}_{1, \mathrm{I}}(t) Q \tilde{\mathscr{L}}_{1, \mathrm{I}}(\tau) \tilde{\rho}(0)\right\} .
\end{aligned}
$$

Although Eq. (85) is local in time, the non-Markovian effects can be explained by the time dependence of $\mathscr{R}(t)$. It's expected to show good performance beyond the typical perturbative regime, as proved for some cases [103]. The expressions of the specific time-local quantum master equation obtained by calculation can be referred to in Ref. [97].

In Sec. B the effect of system-bath entanglement on the EET has been addressed. Besides, this issue could also be explored by the polaron tranformation [104]. Under certain circumstances, the analytical solutions for the EET efficiency can be explicitly given. It is shown that the results by the polaron transformation are consistent with those by the Förster and Redfield theories in the respective limits and interpolate smoothly between them in the intermediate regime.

Beyond the intermediate regime, the small-polaron quantum master equation is accurate in the limits of weak systembath coupling and weak electronic coupling. The smallpolaron quantum master equation has been applied to many studies, such as exploring the role of the relationship between environmental fluctuation and phonon excitation in EET dynamics [105]. And it is also used to study the vibrational contributions to EET in photosynthetic complexes of cryptophyte algae [106]. Specifically, the small-polaron quantum master 
equation can be precisely executed in the incoherent region with larger electron-phonon coupling and in the coherent region with faster bath relaxation. In addition, through the special selection of perturbation terms, the weak electron-phonon coupling limit can also be accurately calculated by the smallpolaron theory [107]. However, due to the perturbation, the accuracy of small-polaron quantum master equation declines in the intermediate-coupling regime.

\section{F. Generalized Bloch-Redfield theory}

In photosynthesis, when a light-harvesting pigment absorbs a photon, the photosynthetic system is activated. The excitation energy is then transferred to the reaction center for subsequent charge separation, resulting in energy trapping. Energy decays during transmission and redistributes through interactions with the protein environment.

For the Debye-form spectral density, $J(\omega)=$ $(2 \hbar / \pi) \lambda \omega D /\left(\omega^{2}+D^{2}\right)$, the spatially-correlated spectral density is $J_{n m}(\omega)=c_{n m} J(\omega)$, where $\lambda$ is the reorganization energy, $D$ is the bath relaxation rate, $c_{m n}$ is the spatial correlation coefficient between sites $m$ and $n$. The time correlation functions corresponding to the Debye spectral density can be expanded using exponential functions [108]

$$
C_{m n}(t)=c_{m n} \sum_{i=0}^{\infty}\left(f_{i}^{r}+\mathrm{i} f_{i}^{i}\right) \mathrm{e}^{-v_{i} t}
$$

where $v_{i}$ is the relaxation rate of the $i$ th bath mode, $f_{i}^{r}$ and $f_{i}^{i}$ are the real and imaginary part of the expansion coefficient, respectively. With the facilitation of auxiliary fields $g_{m ; i}(t)$ at site $m$, the generalized Bloch-Redfield equation for exciton dynamics can be written as [109]

$$
\begin{aligned}
\dot{\rho}(t)= & -\left(L_{\text {sys }}+L_{\text {trap }}\right) \rho(t)-\mathrm{i} \sum_{i=0}^{\infty} \sum_{m}\left[Q_{m}, g_{m ; i}(t)\right], \\
\dot{g}_{m ; i}(t)= & -\left(L_{\text {sys }}+L_{\text {trap }}+v_{i}\right) g_{m ; i}(t) \\
& -\mathrm{i} f_{i}^{r}\left[\Theta_{m}, \rho(t)\right]+f_{i}^{i}\left[\Theta_{m}, \rho(t)\right]_{+},
\end{aligned}
$$

where $\rho$ is the system's reduced density matrix, $Q_{m}=|m\rangle\langle m|$ is the system operator, $\Theta_{m}=\sum_{n} c_{m n} Q_{n}$, and $[A, B]_{+}=A B+$ $B A$ is the anti-commutator. $L_{\text {sys }} \rho=i[\mathbf{H}, \rho] / \hbar$ and $\mathbf{H}_{n m}=$ $\delta_{n, m} \varepsilon_{n}+\left(1-\delta_{n, m}\right) J_{n m}$, where $\varepsilon_{n}$ is the site energy, the offdiagonal element $J_{n m}$ is the dipole-dipole interaction coupling strength between two distinct sites. $\left[L_{\text {trap }}\right]_{n m}=\left(k_{t ; m}+k_{t ; n}\right) / 2$ describes the localization at the charge-separation state, with $k_{\mathrm{t} ; \mathrm{m}}$ the trapping rate at site $m$. The initial value of $g_{m ; i}(t)$ is zero. The auxiliary field represent the memory effect in the dissipative dynamics caused by the interaction with the bath. For the auxiliary fields, it can be considered as an element of the projection operator $Q(1-P)$ in the Nakajima-Zwanzig projection operator technique.

As described earlier, the Redfield theory can be applied to the case of weak dissipation regime, but it can not accurately describe exciton dynamics in the case of intermediate and strong dissipation regimes [33, 110]. The generalized
Bloch-Redfield method proposed in this subsection is applicable to a wide range of dissipation cases and can also predict the correct Förster-rate limit [111].

\section{Discussion and Conclusion}

Generally, the light-harvesting efficiency in the primary process of photosynthetic organisms can reach up to $95 \%$. If we can effectively simulate photosynthesis in nature and learn from it, it will be the most valuable way to solve our current energy problems.

In this paper, we first give a detailed description and summary of the progress in the study of the physical mechanism of EET in photosynthesis. Then, we summarize some theoretical methods for studying incoherent EET in photosynthesis. The Förster theory can be used to calculate the rate of EET, but it considers the transfer of electronic excitation energy from a donor to an acceptor. Therefore, the theory should be generalized when applied to a multichromophoric system. In view of this situation, the generalized Förster theory has been developed. THe Förster theory is applicable to the case where the couplings between pigments are much less than those between the pigments and environment. In this case, the couplings between pigments are regarded as a perturbation. On the contrary, when the couplings between pigments are much greater than those between the pigments and environment, the EET is usually described by the Redfield theory. In this case, the couplings between pigments and environment are regarded as a perturbation. These two theories represent two opposite-limit regions of the coupling strength between pigments and environment. However, because for some intermediate regions, the above two methods are no longer applicable, some new methods and theories are constantly developed, such as the HEOM, which is developed in a non-perturbative manner. The hierarchy equation (48) can describe quantum coherent wavelike motion, and incoherent hopping, and intermediate EET regime in a unified manner. The only disadvantage is that the computational complexity grows exponentially with respect to the system's size and number of exponents in the bath's correlation function. In addition, the quantum master equation by small-polaron transformation has been developed and is used to interpolate between the Redfield and Förster limits by employing the small-polaron transformation. It is based on the second-order perturbative truncation with respect to the renormalized electron-phonon coupling. Furthermore, other methods have been developed, such as, quantum path integrals, MRT and its coherent version CMRT, the generalized Bloch-Redfield theory. These methods are also introduced in this paper.

Conflict of interest The authors declare that they have no conflict of interest.

\section{Acknowledgments}

This work was supported by the National Natural Science Foundation of China (11674033, 11474026, and 11505007). 
Author contributions Gui-Lu Long, Qing Ai, and FuGuo Deng conceived the review topic. Ming-Jie Tao, Na-Na Zhang, and Qing Ai wrote the manuscript in consultation with all the other authors. Na-Na Zhang arranged all the figures. Peng-Yu Wen and Fu-Guo Deng modified the manuscript. All authors contributed to the final manuscript.
[1] N. Lambert, Y.-N. Chen, Y.-C. Cheng, et al., Quantum biology, Nat Phys 9 (2013) 10-18.

[2] Q. Ai, Toward quantum teleporting living objects, Sci Bull 61 (2016) 110-111.

[3] K. Maeda, K. B. Henbest, F. Cintolesi, et al., Chemical compass model of avian magnetoreception, Nature 453 (2008) 387-390.

[4] M. Zapka, D. Heyers, C. M. Hein, et al., Visual but not trigeminal mediation of magnetic compass information in a migratory bird, Nature 461 (2009) 1274-1277.

[5] I. K. Kominis, Quantum Zeno effect explains magneticsensitive radical-ion-pair reactions, Phys Rev E 80 (2009) 056115.

[6] J. M. Cai, G. G. Guerreschi, H. J. Briegel, Quantum control and entanglement in a chemical compass, Phys Rev Lett 104 (2010) 220502.

[7] J. M. Cai, Quantum probe and design for a chemical compass with magnetic nanostructures, Phys Rev Lett 106 (2011) 100501.

[8] E. M. Gauger, E. Rieper, J. J. L. Morton, et al., Sustained quantum coherence and entanglement in the avian compass, Phys Rev Lett 106 (2011) 040503.

[9] T. Ritz, S. Adem, K. Schulten, A model for photoreceptorbased magnetoreception in birds, Biophys J 78 (2000) 707718.

[10] L. P. Yang, Q. Ai, C. P. Sun, Generalized Holstein model for spin-dependent electron-transfer reactions, Phys Rev A 85 (2012) 032707.

[11] H. T. Quan, Z. Song, X. F. Liu, et al., Decay of Loschmidt echo enhanced by quantum criticality, Phys Rev Lett 96 (2006) 140604.

[12] Q. Ai, T. Shi, G. L. Long, et al., Induced entanglement enhanced by quantum criticality, Phys Rev A 78 (2008) 022327.

[13] Q. Ai, Y. D. Wang, G. L. Long, et al., Two mode photon bunching effect as witness of quantum criticality in circuit QED, Sci China Ser G 52 (2009) 1898-1905.

[14] C. Y. Cai, Q. Ai, H. T. Quan, et al., Sensitive chemical compass assisted by quantum criticality, Phys Rev A 85 (2012) 022315.

[15] G. R. Fleming, R. van Grondelle, The primary steps of photosynthesis, Phys Today 2 (1994) 48-55.

[16] J. Deisenhofer, H. Michel, The photosynthetic reaction centre from the purple bacterium Rhodopseudomonas viridis, Biosci Rep 9 (1989) 383-419.

[17] J. Deisenhofer, H. Michel, High-resolution structures of photosynthetic reaction centers, Annu Rev Biophys Biophys Chem 20 (1991) 247-266.

[18] S. Mostame, P. Rebentrost, A. Eisfeld, et al., Quantum simulator of an open quantum system using superconducting qubits: Exciton transport in photosynthetic complexes, New J Phys 14 (2012) 105013.

[19] A. Potočnik, A. Bargerbos, F. A. Y. N. Schröder, et al., Studying light-harvesting models with superconducting circuits, Nat Commun 9 (2018) 904.

[20] M.-J. Tao, M. Hua, Q. Ai, et al., Quantum simulation of clustered photosynthetic light harvesting in a superconducting quantum circuit, arXiv: 1810.05825

[21] Y. C. Cheng, B. Silbey, Coherence in the B800 ring of purple bacteria LH2, Phys Rev Lett 96 (2006) 028103.

[22] L. Xu, Z. R. Gong, M. J. Tao, et al., Artificial light harvesting by Dimerized Möbius Ring, Phys Rev E 97 (2018) 042124.

[23] D. J. Gorman, B. Hemmerling, E. Megidish, et al., Engineering vibrationally assisted energy transfer in a trapped-ion quantum simulator, Phys Rev X 8 (2018) 011038.

[24] A. W. Chin, E. Mangaud, O. Atabek, et al., Coherent quantum dynamics launched by incoherent relaxation in a quantum circuit simulator of a light-harvesting complex, Phys Rev A 97 (2018) 063823

[25] G. S. Engel, T. R. Calhoun, E. L. Read, et al., Evidence for wavelike energy transfer through quantum coherence in photosynthetic systems, Nature 446 (2007) 782-786.

[26] F. Caruso, A. W. Chin, A. Datta, et al., Highly efifcient energy excitation transfer in light-harvesting complexes: The fundamental role of noise-assisted transport, J Chem Phys 113 (2009) 105106.

[27] M. Sarovar, Y. C. Cheng, K. B. Whaley, Environmental correlation effects on excitation energy in photosynthetic light harvesting, Phys Rev E 83 (2011) 011906.

[28] F. Fassioli, A. Olaya-Castro, Distribution of entanglement in light-harvesting complexes and their quantum efficiency, New J Phys 12 (2010) 085006.

[29] A. Eisfeld, J. S. Briggs, Classical master equation for excitonic transport under the influence of an environment, Phys Rev E 85 (2012) 046118.

[30] R. de J. Leon-Montiel, J. P. Torres, Highly efficient noiseassisted energy transport in classical oscillator system, Phys Rev Lett 110 (2013) 218101.

[31] M. Mohseni, P. Rebentrost, S. Lloyd, et al., Environmentassisted quantum walks in photosynthetic energy transfer, J Chem Phys 129 (2008) 174106.

[32] P. Rebentrost, M. Mohseni, I. Kassal, et al., Environmentassisted quantum transport, New J Phys 11 (2008) 033003.

[33] P. Rebentrost, M. Mohseni, A. Aspuru-Guzik, Role of quantum coherence and environmental fluctuations in chromophoric energy transport, J Phys Chem B 113 (2009) 99429947.

[34] J. S. Cao, R. J. Silbey, Optimization of exciton trapping in energy transfer processes, J Phys Chem A 113 (2009) 1382513838.

[35] S. Sachdev, Quantum phase transitions (Cambridge University Press, Cambridge, 1999).

[36] M. J. Tao, Q. Ai, F. G. Deng, et al., Proposal for probing energy transfer pathway by single-molecule pump-dump experiment, Sci Rep 6 (2016) 27535.

[37] W. M. Zhang, T. Meier, V. Chernyak, et al., Exciton-migration and three-pulse femtosecond optical spectroscopies of photosynthetic antenna complexes, J Chem Phys 108 (1998) 77637774.

[38] A. Olaya-Castro, C. F. Lee, F. F. Olsen, et al., Efficiency of energy transfer in a light-harvesting system under quantum coherence, Phys Rev B 78 (2008) 085115.

[39] X. Hu, T. Ritz, A. Damjanovic, et al., Pigment organization 
and transfer of electronic excitation in the photosynthetic unit of purple bacteria, J Phys Chem B 101 (1997) 3854-3871.

[40] Q. S. Tan, Energy and information transfer in simple photosynthetic model and coupled quantum dot system (Hunan Normal University, 2014).

[41] S. Yang, D. Z. Xu, Z. Song, et al., Dimerization-assisted energy transport in light-harvesting complexes, J Chem Phys 132 (2010) 234051.

[42] X. T. Liang, Excitation energy transfer: Study with nonMarkovian dynamics, Phys Rev E 82 (2010) 051918.

[43] G. D. Scholes, X. J. Jordanides, G. R. FlerIling, Adapting the Förster theory of energy transfer for modeling dynamics in aggregated molecular assemblies, J Phys Chem B 105 (2001) 1640-1651.

[44] M. Yang, G. R. Fleming, Influence of phonons on exciton transfer dynamics: comparison of the Redfield, Förster, and modified Redfield equations, Chem Phys 275 (2002) 355-372.

[45] S. Jang, Y. J. Jung, R. J. Silbey, Nonequilibrium generalization of Förster-Dexter theory for excitation energy transfer, Chem Phys 275 (2002) 319-332.

[46] A. Ishizaki, G. R. Fleming, On the adequacy of the Redfield equation and related approaches to the study of quantum dynamics in electronic energy transfer, J Chem Phys 130 (2009) 234110.

[47] V. May, O. Kühn, Charge and Energy Transfer Dynamics in Molecular Systems, 2nd ed. (Wiley-VCH Verlag, Berlin, 2004).

[48] S. Mukamel, Principles of Nonlinear Optics and Spectroscopy, (Oxford University Press, USA, 1999).

[49] S. Jang, M. D. Newton, R. J. Silbey, Multichromophoric Förster resonance energy transfer, Phys Rev Lett 92 (2004) 218301.

[50] Q. Ai, T. C. Yen, B. Y. Jin, et al., Clustered geometries exploiting quantum coherence effects for efficient energy transfer in light harvesting, J Phys Chem Lett 4 (2013) 2577-2584.

[51] H. Sumi, Theory on rates of excitation-energy transfer between molecular aggregates through distributed transition dipoles with application to the antenna system in bacterial photosynthesis, J Phys Chem B 103 (1999) 252-260.

[52] J. Ma, J. S. Cao, Förster resonance energy transfer, absorption and emission spectra in multichromophoric systems. I. Full cumulant expansions and system-bath entanglement, J Chem Phys 142 (2015) 094106.

[53] J. Ma, J. Moix, J. S. Cao, Förster resonance energy transfer, absorption and emission spectra in multichromophoric systems. II. Hybrid cumulant expansion, J Chem Phys 142 (2015) 094107.

[54] J. Moix, J. S. Cao, Förster resonance energy transfer, absorption and emission spectra in multichromophoric systems. III. Exact stochastic path integral evaluation, J Chem Phys 142 (2015) 094108.

[55] V. Novoderezhkin, R.van Grondelle, Physical origins and models of energy transfer in photosynthetic light-harvesting, Phys Chem Chem Phys 12 (2010) 7352-7365.

[56] V. Novoderezhkin, R.van Grondelle, Spectra and Dynamics in the B800 Antenna: Comparing Hierarchical Equations, Redfield and Förster Theories, J Phys Chem B 117 (2013) 1107611090.

[57] H . Lee, Y. C. Cheng, G. R. Fleming, Coherence dynamics in photosynthesis: Protein protection of excitonic coherence, Science 316 (2007) 1462-1465.

[58] E. Collini, C. Y. Wong, K. E. Wilk, et al., Coherently wired light-harvesting in photosynthetic marine algae at ambient temperature, Nature 463 (2010) 644-647.
[59] R. Hildner, D. Brinks, J. B. Nieder, et al., Quantum coherent energy transfer over varying pathways in single lightharvesting complexes, Science 340 (2013) 1448-1451.

[60] H. P. Breuer, A. Ma, F. Petruccione, Time-Local master equations: influence functional and cumulant expansion, In: Leggett A.J., Ruggiero B., Silvestrini P. (eds) Quantum Computing and Quantum Bits in Mesoscopic Systems, (2004) 263271.

[61] A. O. Caldeira, A. J. Leggett, Path integral approach to quantum Brownian motion, Physica 121A (1983) 587-616.

[62] H. Grabert, P. Schramm, G. L. Ingold, Quantum Brownian motion: The functional integral approach, Phys Rep 168 (1988) 115-207.

[63] A. Ishizaki, G. Fleming, Unified treatment of equation coherent and incoherent hopping dynamics in electronic energy transfer: Reduced hierarchy equation approach, J Chem Phys 130 (2009) 234111.

[64] Z. F. Tang, X. L. Ouyang, Z. H. Gong, et al., Extended hierarchy equation of motion for the spin-boson model, J Chem Phys 143 (2015) 224112.

[65] H. Liu, L. L. Zhu, S. M. Bai, et al., Reduced quantum dynamics with arbitrary bath spectral densities: Hierarchical equations of motion based on several different bath decomposition schemes, J Chem Phys 140 (2014) 134106.

[66] M. Schröder, M. Schreiber, Reduced dynamics of coupled harmonic and anharmonic oscillators using higher-order perturbation theory, J Chem Phys 126 (2007) 114102.

[67] Y. J. Yan, Theoretical method for open quantum systems: Progresses and perspectives, J Uni Sci Tech Chn 43 (2013) 861869.

[68] J. Xu, H. D. Zhang, R. X. Xu, et al., Correlated driving and dissipation in two-dimensional spectroscopy, J Chem Phys 138 (2013) 024106.

[69] L. P. Chen, R. H. Zheng, Y. Y. Jing, et al., Simulation of the two-dimensional electronic spectra of the Fenna-MatthewsOlson complex using the hierarchical equations of motion method, J Chem Phys 138 (2011) 194508.

[70] X. Zheng, J. S. Jin, Y. J. Yan, Dynamic electronic response of a quantum dot driven by time-dependent voltage, J Chem Phys 129 (2008) 184112.

[71] X. Zheng, J. S. Jin, Y. J. Yan, Dynamic Coulomb blockade in single-lead quantum dots, New J Phys 10 (2008) 093016.

[72] X. Zheng, J. Y. Luo, J. S. Jin, et al., Complex non-Markovian effect on time-dependent quantum transport, J Chem Phys 130 (2009) 124508.

[73] X. Zheng, J. S. Jin, S. Welack, et al., Numerical approach to time-dependent quantum transport and dynamical Kondo transitio, J Chem Phys 130 (2009) 164708.

[74] R. P. Feynman, F. L. Vernon, The theory of a general quantum system interacting with a linear dissipative system, Ann Phys 24 (1963) 118-173.

[75] U. Weiss, Quantum Dissipative Systems (World Scientific Publishing Company, Singapore, 2009).

[76] H. Kleinert, Path Integrals in Quantum Mechanics, Statistics, Polymer Physics, and Financial Markets (World Scientific Publishing, Singapore, 2009).

[77] Y. Tanimura, R. Kubo, Time evolution of a quantum system in contact with a nearly Gaussian-Markovian noise bath, J Phys Soc Jpn 58 (1989) 101-114.

[78] Y. Tanimura, Nonperturbative expansion method for a quantum system coupled to a harmonicoscillator bath, Phys Rev A 41 (1990) 6676-6687.

[79] R. X. Xu, P. Cui, X. Q. Li, et al., Exact quantum master equation via the calculus on path integrals, J Chem Phys 122 (2005) 
041103.

[80] R. X. Xu, Y. J. Yan, Dynamics of quantum dissipation systems interacting with bosonic canonical bath: Hierarchical equations of motion approach, Phys Rev E 75 (2007) 031107.

[81] J. S. Jin, X. Zheng, Y. J. Yan, Exact dynamics of dissipative electronic systems and quantum transport: Hierarchical equations of motion approach, J Chem Phys 128 (2008) 234703.

[82] J. S. Jin, S. Welack, J. Y. Luo, et al., Dynamics of quantum dissipation systems interacting with fermion and boson grand canonical bath ensembles: Hierarchical equations of motion approach, J Chem Phys 126 (2007) 134113.

[83] J. Hu, R. X. Xu, Y. J. Yan, Communication: Pade spectrum decomposition of Fermi function and Bose function, J Chem Phys 133 (2010) 101106.

[84] J. Hu, M. Luo, F. Jiang, et al., Pade spectrum decompositions of quantum distribution functions and optimal hierarchical equations of motion construction for quantum open systems, J Chem Phys 134 (2011) 244106.

[85] A. Ishizaki, G. R. Fleming, Theoretical examination of quantum coherence in a photosynthetic system at physiological temperature, Proc Natl Acad Sci USA 106 (2009) 1725517260.

[86] T. Renger, V. May, O. Kuhn, Ultrafast excitation energy dynamics in photosynthetic pigment-protein complexes, Phys Rep 343 (2001) 137-254.

[87] Y. C. Cheng, G. R. Fleming, Dynamics of light harvesting in photosynthesis, Annu Rev Phys Chem 60 (2009) 241-262.

[88] B. X. Wang, M. J. Tao, Q. Ai, et al., Efficient quantum simulation of photosynthetic light harvesting, npj Quantum Inf 4 (2018) 52.

[89] W. Jiang, F. Z. Wu, G. J. Yang, Non-Markovian entanglement dynamics of open quantum systems with continuous measurement feedback, Phys Rev A 98 (2018) 052134.

[90] M. Thorwart, P. Reimann, P. Jung, et al., Quantum hysteresis and resonant tunneling in bistable systems, Chem Phys 235 (1998) 61-80.

[91] N. Makri, Numerical path integral techniques for long time dynamics of quantum dissipative systems, J Math Phys 36 (1995) 2430-2457.

[92] N. Makri, D. E. Makarov, Tensor propagator for iterative quantum time evolution of reduced density matrices. II. Numerical methodology, J Chem Phys 102 (1995) 4611-4618.

[93] Y.-H. Hwang-Fu, W. Chen, et al., A coherent modified Redfield theory for excitation energy transfer in molecular aggregates, Chem Phys 447 (2015) 46-53.

[94] Y. Chang, Y. C. Cheng, On the accuracy of coherent modified Redfield theory in simulating excitation energy transfer dynamics, J Chem Phys 142 (2015) 034109.

[95] H. P. Breuer, F. Petruccione, The Theory of Open Quantum Systems, (Oxford University Press, New York, 2002).
[96] Q. Ai, Y. J. Fan, B. Y. Jin, et al., An efficient quantum jump method for coherent energy transfer dynamics in photosynthetic systems under the influence of laser fields, New J Phys 16 (2014) 053033.

[97] S. Jang, Y. C. Cheng, D. R. Reichman, et al., Theory of coherent resonance energy transfer, J Chem Phys 129 (2008) 101104.

[98] T. Holstein, Studies of polaron motion: Part I. The molecularcrystal model, Ann Phys (N Y) 8 (1959) 325-342.

[99] R. Silbey, R. A. Harris, Variational calculation of the dynamics of a two level system interacting with a bath, J Chem Phys 80 (1984) 2615-2617.

[100] R. A. Harris, R. Silbey, Variational calculation of the tunneling system interacting with a heat bath. II. Dynamics of an asymmetric tunneling system, J Chem Phys 83 (1985) 1069-1074.

[101] S. Rackovsky, R. Silbey, Electronic energy transfer in impure solids, Mol Phys 25 (1973) 61-72.

[102] B. Jackson, R. Silbey, On the calculation of transfer rates between impurity states in solids, J Chem Phys 78 (1983) 41934196.

[103] M. A. Palenberg, R. J. Silbey, C. Warns, et al., Local and nonlocal approximation for a simple quantum system, J Chem Phys 114 (2001) 4386-4389.

[104] D. Z. Xu, J. S. Cao, Non-canonical distribution and nonequilibrium transport beyond weak system-bath coupling regime: A polaron transformation approach, Front Phys 11 (2016) 110308.

[105] A. Nazir, Correlation-dependent coherent to incoherent transitions in resonant energy transfer dynamics, Phys Rev Lett 103 (2013) 146404.

[106] A. Kolli, E. J. ÓReilly, G. D. Scholes, et al., The fundamental role of quantized vibrations in coherent light harvesting by cryptophyte algae, J Chem Phys 137 (2012) 174109.

[107] H. T. Chang, P. P. Zhang, Y. C. Cheng, Criteria for the accuracy of small polaron quantum master equation in simulating excitation energy transfer dynamics, J Chem Phys 139 (2013) 224112.

[108] S. Jang, J. Cao, R. J. Silbey, Fourth-order quantum master equation and its Markovian bath limit, J Chem Phys 116 (2002) 2705-2717

[109] J. L. Wu, F. Liu, Y. Shen, et al., Efficient energy transfer in light-harvesting systems, I: optimal temperature, reorganization energy and spatial-temporal correlations, New J Phys 12 (2010) 105012.

[110] M. Grover, R. J. Silbey, Exciton migration in molecular crystals, J Chem Phys 54 (1971) 4843-4851.

[111] J. S. Cao, A phase-space study of Bloch-Redfield theory, J Chem Phys 107 (1997) 3204-3209. 